\title{
On the spectrum of linear combinations of finitely many diagonalizable matrices that mutually commute
}

https://doi.org/10.1515/spma-2020-0138

Received March 2, 2021; accepted May 15, 2021

\begin{abstract}
We propose an algorithm, which is based on the method given by Kişi and Özdemir in [Math Commun, 23 (2018) 61], to handle the problem of when a linear combination matrix $X=\sum_{i=1}^{m} c_{i} X_{i}$ is a matrix such that its spectrum is a subset of a particular set, where $c_{i}, i=1,2, \ldots, m$, are nonzero scalars and $X_{i}$, $i=1,2, \ldots, m$, are mutually commuting diagonalizable matrices. Besides, Mathematica implementation codes of the algorithm are also provided. The problems of characterizing all situations in which a linear combination of some special matrices, e.g. the matrices that coincide with some of their powers, is also a special matrix can easily be solved via the algorithm by choosing of the spectra of the matrices $X$ and $X_{i}$, $i=1,2, \ldots, m$, as subsets of some particular sets. Nine of the open problems in the literature are solved by utilizing the algorithm. The results of the four of them, i.e. cubicity of linear combinations of two commuting cubic matrices, quadripotency of linear combinations of two commuting quadripotent matrices, tripotency of linear combinations of three mutually commuting tripotent matrices, and tripotency of linear combinations of four mutually commuting involutive matrices, are presented explicitly in this work. Due to the length of their presentations, the results of the five of them, i.e. quadraticity of linear combinations of three or four mutually commuting quadratic matrices, cubicity of linear combinations of three mutually commuting cubic matrices, quadripotency of linear combinations of three mutually commuting quadripotent matrices, and tripotency of linear combinations of four mutually commuting tripotent matrices, are given as program outputs only. The results obtained are extensions and/or generalizations of some of the results in the literature.
\end{abstract}

Keywords: algorithm, diagonalizable matrices, spectrum

MSC: 68W01, 15A27, 15A06, 15B99

\section{Introduction}

Let $\mathbb{N}, \mathbb{C}$, and $\mathbb{C}_{n}$ denote the sets of natural numbers, complex numbers, and $n \times n$ complex matrices, respectively. $\mathbf{0}$ and $I_{n}$ will be a zero matrix of appropriate size and the identity matrix of order $n$, respectively. The symbols $\sigma(A), A^{*}, A^{\dagger}$, and $A^{\sharp}$ will stand for spectrum, conjugate transpose, Moore-Penrose inverse, and group inverse of $A \in \mathbb{C}_{n}$, respectively. For details on generalized inverses of matrices see [10]. A matrix $A \in \mathbb{C}_{n}$ is said to be idempotent (projector), orthogonal projector, involutory, tripotent, quadripotent, generalized projector, hypergeneralized projector, group involutory, and generalized involutive if $A^{2}=A, A^{2}=A=A^{*}, A^{2}=I_{n}$, $A^{3}=A, A^{4}=A, A^{2}=A^{*}, A^{2}=A^{\dagger}, A=A^{\sharp}$, and $A=A^{\dagger}$, respectively.

\footnotetext{
^Corresponding Author: Emre Kişi: Department of Mathematics, Sakarya University, Sakarya TR54187 Turkey, E-mail: ekisi@sakarya.edu.tr

Murat Sarduvan: Department of Mathematics, Sakarya University, Sakarya TR54187 Turkey, E-mail: msarduvan@sakarya.edu.tr

Halim Özdemir: Department of Mathematics, Sakarya University, Sakarya TR54187 Turkey, E-mail: hozdemir@sakarya.edu.tr Nurgül Kalaycı: Department of Mathematics, Sakarya University, Sakarya TR54187 Turkey, E-mail: nrglklyc@hotmail.com
} 
Let $k \in \mathbb{N}$ and $\alpha, \beta, \gamma \in \mathbb{C}$ such that $\alpha \neq \beta, \alpha \neq \gamma, \beta \neq \gamma$, and $k \geq 2$. Then a matrix $A \in \mathbb{C}_{n}$ is said to be $\{\alpha, \beta\}$-quadratic, $\{\alpha, \beta, \gamma\}$-cubic, $k$-potent, $k$-generalized projector, $k$-hypergeneralized projector, and $\{k\}$-group periodic if $\left(A-\alpha I_{n}\right)\left(A-\beta I_{n}\right)=\mathbf{0},\left(A-\alpha I_{n}\right)\left(A-\beta I_{n}\right)\left(A-\gamma I_{n}\right)=\mathbf{0}, A^{k}=A, A^{k}=A^{*}, A^{k}=A^{\dagger}$, and $A^{k-1}=A^{\sharp}$, respectively.

Some of these special matrices mentioned above play an important role in applied sciences. For instance, idempotent and tripotent matrices are used in statistical theory [18, Section 12.4] and quadratic forms [9], involutory matrices are used in quantum mechanics [1, p. 495] and cryptography [20, Section 6.11], and $k$ potent matrices are used in digital image encryption [28]. Further examples can be found in [13, 17, 25].

The problems of characterizing all situations in which a linear combination of some special matrices is also a special matrix have been studied by many authors in recent years. The results in the literature are summarized in the following tables. Linear combinations considered in the literature are as follows:

$$
X=c_{1} X_{1}+c_{2} X_{2}
$$

and

$$
X=c_{1} X_{1}+c_{2} X_{2}+c_{3} X_{3},
$$

where $X_{j} \in \mathbb{C}_{n}$ and $c_{j} \in \mathbb{C} \backslash\{0\}, j=1,2,3$. The results concerning linear combinations of the form (1) and linear combinations of the form (2) are listed in Table 1 and Table 2, respectively. Please note that $i \in\{1,2\}$ in Table 1 and Table 2 and $j \in\{1,2,3\}$ in Table 2.

Table 1: Summary of the results in which the linear combinations of the form (1) are considered

\begin{tabular}{lll}
\hline & $X_{1} X_{2}=X_{2} X_{1}$ & $X_{1} X_{2} \neq X_{2} X_{1}$ \\
\hline$X^{2}=X$ & $X_{i}^{2}=X_{i}[2]$ & $X_{i}^{2}=X_{i}$ [2] \\
& $X_{1}^{2}=X_{1}$ and $X_{2}^{3}=X_{2}[6]$ & $X_{1}^{2}=X_{1}$ and $X_{2}^{3}=X_{2}[6]$ \\
& $X_{i}^{3}=X_{i}[24]$ & \\
& $X_{1}^{2}=X_{1}$ and $X_{2}^{k}=X_{2}[11]$ & $X_{1}^{2}=X_{1}$ and $X_{2}^{k}=X_{2}[14]$ \\
& $X_{i}^{2}=I_{n}[25]$ & $X_{i}^{2}=I_{n}[25]$ \\
& $X_{1}^{3}=X_{1}$ and $X_{2}^{2}=I_{n}[29]$ & $X_{1}^{3}=X_{1}$ and $X_{2}^{2}=I_{n}[29]$ \\
\hline$X^{2}=I_{n}$ & $X_{i}^{2}=X_{i}[25]$ & $X_{i}^{2}=X_{i}[25]$ \\
& $X_{i}^{2}=I_{n}[25]$ & $X_{i}^{2}=I_{n}[25]$ \\
& $X_{i}^{3}=X_{i}[25]$ & \\
$X^{\sharp}=X$ & $X_{1}^{3}=X_{1}$ and $X_{2}^{3}=I_{n}[29]$ & $X_{1}^{3}=X_{1}$ and $X_{2}^{3}=I_{n}[29]$ \\
$X^{\dagger}=X$ & $X_{i}^{2}=X_{i}[4,16]$ & $X_{i}^{2}=X_{i}[4,16]$ \\
$X^{3}=X$ & $X_{i}^{\dagger}=X_{i}[23]$ & $X_{i}^{\dagger}=X_{i}[23]$ \\
\hline$X^{2}=X^{*}$ & $X_{i}^{2}=I_{n}[25]$ & \\
$X^{2}=X^{\dagger}$ & $X_{i}^{3}=X_{i}[5,24]$ & \\
$X^{k}=X$ & $X_{i}^{2}=X_{i}^{*}[3,12]$ & \\
$X^{k}=X^{*}$ & $X_{i}^{2}=X_{i}^{\dagger}[8]$ & \\
$X^{k-1}=X^{\sharp}$ & $X_{i}^{2}=I_{n}[26]$ & \\
$\left(X-\alpha I_{n}\right)\left(X-\beta I_{n}\right)=\mathbf{0}=X_{i}^{*}[3,12]$ \\
\hline
\end{tabular}

In literature, there are more results about commuting matrices, since the characterization problems aforementioned can be solved relatively easily with the assumption of commutativity. However, even under the assumption of commutativity, this kind of problems becomes more compelling as the number of matrices in the linear combination increases. It is seen from the Table 1 and Table 2 that the linear combinations of 
Table 2: Summary of the results in which the linear combinations of the form (2) are considered

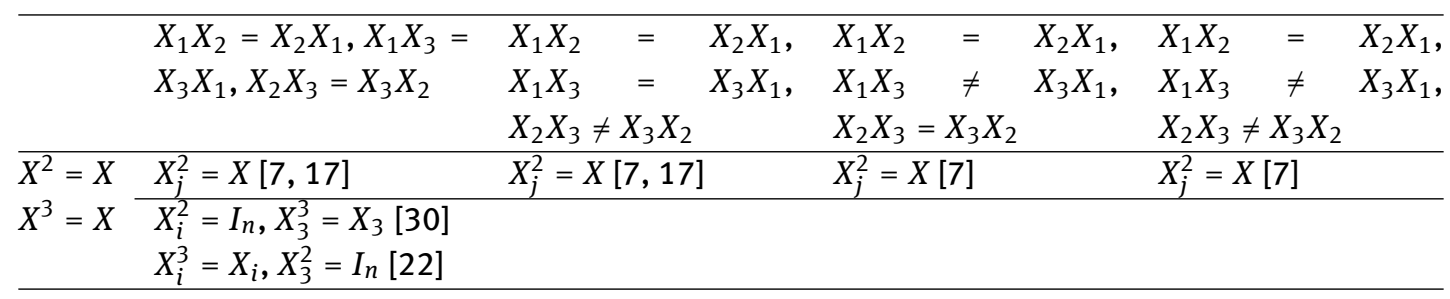

two matrices have been studied for many different types of special matrices, while the linear combinations of three matrices have been considered just for idempotent and tripotent matrices.

Under the assumption of mutual commutativity, the problems of characterizing all situations in which a linear combination of some special matrices is also a special matrix are equivalent to the problem of when a linear combination of diagonalizable matrices whose spectra are subsets of some particular sets is a matrix such that its spectrum is the subset of a particular set. For instance, the problem of tripotency of linear combinations of two commuting tripotent matrices solved in [5] is equivalent to the problem when a linear combination matrix $T=c_{1} T_{1}+c_{2} T_{2}$ is a matrix such that $\sigma(T) \subseteq\{1,-1,0\}$, where $T_{1}$ and $T_{2}$ are commuting diagonalizable matrices such that $\sigma\left(T_{1}\right) \subseteq\{1,-1,0\}$ and $\sigma\left(T_{2}\right) \subseteq\{1,-1,0\}$. Two common properties of special matrices listed in Table 1 and Table 2 are that they are diagonalizable and their spectra are subsets of some particular sets.

In [22], Kişi and Özdemir proposed a combinatorial method to handle the problem of characterizing all situations in which the spectrum of the linear combination of finitely many mutually commuting diagonalizable matrices is a subset of a particular set. The method is based on solving the systems of linear equations whose coefficients are taken from the spectra of the matrices in the linear combination. This new method makes it possible to solve such characterization problems for the linear combinations of finitely many special matrices. However, as the number of the matrices in the linear combinations increases, the number of linear systems that need to be solved increases exponentially, and at some point, the number of required calculations reach a number that cannot be done by hand.

In the present work, an algorithm is developed based on the method given in [22]. Besides, Mathematica 8.0 implementation codes of the algorithm are also provided (see DAS). Thus, the problems of characterizing the situations in which the linear combinations of mutually commuting special matrices is also a special matrix have become solvable by means of computers. Moreover, nine of the open problems which are listed in the following are solved by utilizing the algorithm developed in this work:

1. cubicity of linear combinations of two commuting cubic matrices,

2. quadripotency of linear combinations of two commuting quadripotent matrices,

3. tripotency of linear combinations of three tripotent matrices that mutually commute,

4. tripotency of linear combinations of four involutive matrices that mutually commute,

5. quadraticity of linear combinations of three quadratic matrices that mutually commute,

6. cubicity of linear combinations of three cubic matrices that mutually commute,

7. quadripotency of linear combinations of three quadripotent matrices that mutually commute,

8. quadraticity of linear combinations of four quadratic matrices that mutually commute,

9. tripotency of linear combinations of four tripotent matrices that mutually commute.

For the sake of shortness the results of the first four problems are presented explicitly in this work and the rest of them are given as program outputs only (see DAS). The results obtained in the first problem are a generalization of the results given for tripotent matrices in [5]. The results obtained in the second problem cover those established for the generalized and hypergeneralized projectors in $[3,8,12]$. The results obtained in the third problem is an expanding of the results given in [5] and cover those established in [22,30]. 


\section{Algorithm}

In this section, firstly, the combinatorial method which is given in [22] for characterizing the spectrum of the linear combinations of finitely many diagonalizable matrices that mutually commute is reminded, and then the algorithm based on the method is given.

\subsection{The method}

Let $X_{i} \in \mathbb{C}_{n}, i=1,2, \ldots, m$, be mutually commuting diagonalizable matrices such that $\sigma\left(X_{i}\right) \subseteq$ $\left\{\lambda_{1}^{i}, \lambda_{2}^{i}, \ldots, \lambda_{n_{i}}^{i}\right\}, n_{i} \leq n$ and $i=1,2, \ldots, m$. The problem considered is to characterize all situations in which linear combination of the form

$$
c_{1} X_{1}+c_{2} X_{2}+\cdots+c_{m} X_{m}=X
$$

is a matrix such that $\sigma(X) \subseteq\left\{\lambda_{1}, \lambda_{2}, \ldots, \lambda_{n_{x}}\right\}$, where $n_{x} \leq n$ and $c_{i} \in \mathbb{C} \backslash\{0\}, i=1,2, \ldots, m$. Since the matrices $X_{i}, i=1,2, \ldots, m$, are diagonalizable and mutually commute, they are simultaneously diagonalizable [21, Theorem 1.3.21], and clearly the matrix $X$ is also simultaneously diagonalizable with the matrices $X_{i}, i=$ $1,2, \ldots, m$. Thus, there is a nonsingular matrix $S \in \mathbb{C}_{n}$ such that

$$
c_{1} S^{-1} X_{1} S+c_{2} S^{-1} X_{2} S+\cdots+c_{m} S^{-1} X_{m} S=S^{-1} X S,
$$

or equivalently

$$
c_{1} \Lambda_{1}+c_{2} \Lambda_{2}+\cdots+c_{m} \Lambda_{m}=\Lambda,
$$

where $\Lambda_{i}$ and $\Lambda$ are the corresponding diagonal matrices of the matrices $X_{i}$ and $X$, respectively. Without loss of generality, including multiplicities of eigenvalues, the explicit form of the linear combination (4) can be written as follows:

$$
c_{1}\left(\begin{array}{ccc}
\lambda_{1}^{1} & & \mathbf{0} \\
& \ddots & \\
\mathbf{0} & & \lambda_{n_{1}}^{1}
\end{array}\right)+\cdots+c_{m}\left(\begin{array}{ccc}
\lambda_{1}^{m} & & \mathbf{0} \\
& \ddots & \\
\mathbf{0} & & \lambda_{n_{m}}^{m}
\end{array}\right)=\left(\begin{array}{ccc}
\lambda_{1} & & \mathbf{0} \\
& \ddots & \\
\mathbf{0} & & \lambda_{n_{x}}
\end{array}\right) .
$$

It is seen from equation (5) that the characterization of the linear combination (3) is based on solving the systems of linear equations, whose unknowns are the scalars $c_{i}$; hereafter, systems of linear equations will be shortly called linear systems. Therefore, firstly, all possible left-hand sides of linear equations and multiple right-hand sides containing all possible constant terms should be created. Then, left-hand sides of linear systems need to be formed by considering these left-hand sides of linear equations combinatorially. The results are obtained by solving all of the linear systems which are formed by combining the left-hand sides of linear systems with the appropriate sized multiple right-hand sides. The details are in the following.

The coefficients of the unknowns $c_{i}$ are chosen from the corresponding sets $\sigma\left(X_{i}\right), i=1,2, \ldots, m$. Thus, there are $n_{i}$ different coefficients for every unknown $c_{i}, i=1,2, \ldots, m$, and by the product rule there are $n_{1} n_{2} \cdots n_{m}$ possible left-hand sides of the linear equations. Since there will be at most $m$ linearly independent equations, it is sufficient to consider only underdetermined and square linear systems. Left-hand sides of the linear systems are formed by considering these $n_{1} n_{2} \cdots n_{m}$ different left-hand sides of the linear equations first one-by-one, then two-by-two, and by continuing the process finally $m$-by- $m$. Hence, the number of linear systems that need to be solved is $\left(\begin{array}{c}n_{1} n_{2} \cdots n_{m} \\ 1\end{array}\right)+\left(\begin{array}{c}n_{1} n_{2} \cdots n_{m} \\ 2\end{array}\right)+\cdots+\left(\begin{array}{c}n_{1} n_{2} \cdots n_{m} \\ m\end{array}\right)$. Constant terms of the multiple righthand sides are chosen from the set $\sigma(X)$. The sizes of the multiple right-hand sides of the linear systems having one linear equation, two linear equations,..., and $m$ linear equations are $1 \times n_{x}, 2 \times n_{x}^{2}, \ldots$, and $m \times n_{x}^{m}$, respectively. Note that there are diagonal matrices $\Lambda_{i}, i=1,2, \ldots, m$, corresponding to each left-hand side of the linear systems. For instance, corresponding diagonal matrices of the linear system

$$
\left(\begin{array}{cccc}
\lambda_{1}^{1} & \lambda_{3}^{2} & \cdots & \lambda_{8}^{m} \\
\lambda_{2}^{1} & \lambda_{5}^{2} & \cdots & \lambda_{13}^{m}
\end{array}\right)\left(\begin{array}{c}
c_{1} \\
c_{2} \\
\vdots \\
\vdots \\
c_{m}
\end{array}\right)=\left(\begin{array}{cccc}
\lambda_{1} & \lambda_{1} & \cdots & \lambda_{n_{x}} \\
\lambda_{1} & \lambda_{2} & \cdots & \lambda_{n_{x}}
\end{array}\right)
$$


are $\Lambda_{1}=\lambda_{1}^{1} I_{r_{1}} \oplus \lambda_{2}^{1} I_{r_{2}}, \Lambda_{2}=\lambda_{3}^{2} I_{r_{1}} \oplus \lambda_{5}^{2} I_{r_{2}}, \cdots$, and $\Lambda_{m}=\lambda_{8}^{m} I_{r_{1}} \oplus \lambda_{13}^{m} I_{r_{2}}$, where the symbol $\oplus$ denotes the direct sum of matrices.

The characterization of the linear combination (3) is obtained by determining the conditions on the scalars $c_{i}$ and the matrices $X_{i}, i=1,2, \ldots, m$. Solutions of the linear systems give the conditions on the scalars $c_{i}, i=1,2, \ldots, m$. There are two situations in determining the conditions on the matrices $X_{i}, i=1,2, \ldots, m$. In the linear systems having infinitely many solutions, the conditions on the matrices are obtained from the row reduced echelon form of the corresponding left-hand side of the linear systems. In the linear systems whose set of solutions consists of unique solutions, there are two situations according to types of the matrices considered. If the problem considered is in the form of $k$-potency of linear combinations of $k$-potent matrices, firstly a general equality for the matrices is calculated by applying the conditions of the problem to the linear combination. Then the conditions on the matrices can be obtained by putting any solution of the results in this general equality. To avoid obtaining the same conditions on the matrices, try to put the solution not used before. (In this work, it has been preferred to put the first solution; and if it is used previously, then the second solution has been put and thus the conditions on the matrices have been obtained by progressing in this way). If the problem considered is to characterize the linear combinations of special matrices which are not $k$-potent matrix but spectra's are subsets of some particular sets, e.g. the problem of quadraticity or cubicity of linear combinations of quadratic or cubic matrices, then corresponding diagonal matrices $\Lambda_{i}, i=1,2, \ldots, m$, of the linear systems are written explicitly as the conditions on the matrices.

It is noteworthy to mention the effects of some special cases of the spectra $\sigma\left(X_{i}\right), i=1,2, \ldots, m$, on the linear systems. If the element zero " 0 " is included by some spectra $\sigma\left(X_{i}\right), i=1,2, \ldots, m$, then left-hand sides of the linear systems occur some of whose columns are zero vectors. Since the matrices corresponding to these zero columns are zero matrices, such left-hand sides will not be considered in the process of forming linear systems. If all of the spectra $\sigma\left(X_{i}\right), i=1,2, \ldots, m$, contain zero, then a left-hand side of the linear equation appears such that

$$
0 c_{1}+0 c_{2}+\cdots+0 c_{m}
$$

Obviously, it is meaningless to consider left-hand side (6) in the process of forming linear systems. Hence, the number of linear systems that need to be solved decreases to $\left(\begin{array}{c}n_{1} n_{2} \cdots n_{m}-1 \\ 1\end{array}\right)+\left(\begin{array}{c}n_{1} n_{2} \cdots n_{m}-1 \\ 2\end{array}\right)+\cdots+\left(\begin{array}{c}n_{1} n_{2} \cdots n_{m}-1 \\ m\end{array}\right)$. When the intersections of the spectra contain more than two elements, linear systems that have a permutation between their solutions arise, and the spectra containing some eigenvalues with their opposite signs lead to the existence of linear systems whose solutions are minus times of each other. Moreover, if all spectra including $\sigma(X)$ contain all eigenvalues with their opposite signs, then there exists a minus times of each linear equation. In that case, in the process of forming linear systems it is sufficient to get one of these two left-hand sides, which are minus times of each other. Hence, the numbers of the left-hand sides are halved, and thus the number of linear systems that need to be solved decreases to $\left(\begin{array}{c}n_{1} n_{2} \cdots n_{m} / 2 \\ 1\end{array}\right)+\left(\begin{array}{c}n_{1} n_{2} \cdots n_{m} / 2 \\ 2\end{array}\right)+\cdots+\left(\begin{array}{l}n_{1} n_{2} \cdots n_{m} / 2 \\ m\end{array}\right)$.

The results which have a permutation between their solutions, or the results whose solutions are minus times of each other are similar results. A characteristic of the characterization of the linear combination (3) is obtained by combining such similar results.

Remark 1. As pointed out previously, it is unnecessary to consider left-hand side (6) in the process of forming linear systems and it is sufficient to get one of the two left-hand sides, which are minus times of each other, while forming linear systems in the case when all the spectra contain all the eigenvalues with their opposite signs. However, even though left-hand side (6) is not considered and one of two left-hand sides, which are minus times of each other, is considered while forming linear systems, assuming that there are also the corresponding blocks of the left-hand sides which are not considered in the corresponding diagonal matrices of linear systems makes us obtain the results for more general matrices.

Remark 2. One who wants to consider the problem only for the linearly independent matrices can omit to solve the underdetermined linear systems. Thus, the number of linear systems that need to be solved decreases to $\left(\begin{array}{c}n_{1} n_{2} \cdots n_{m} \\ m\end{array}\right)$. 
Remark 3. As stated before, there are diagonal matrices $\Lambda_{i}, i=1,2, \ldots, m$, corresponding to each linear system. Since in the method only the underdetermined and square linear systems are considered, the results are obtained for the diagonal matrices which have at most $m$ blocks. If the results are desired for the diagonal matrices which have more than $m$ blocks, then the problem of solving overdetermined linear systems is encountered. Solutions of the overdetermined linear systems can be obtained by intersecting the sets of the solutions of their sub-square linear systems, which have already been solved.

\subsection{The algorithm}

In this section, an algorithm which gives the characterization of the linear combination (3) and based on the method presented in the previous section is given. Moreover, Mathematica 8.0 implementation codes of the algorithm are also provided (see DAS).

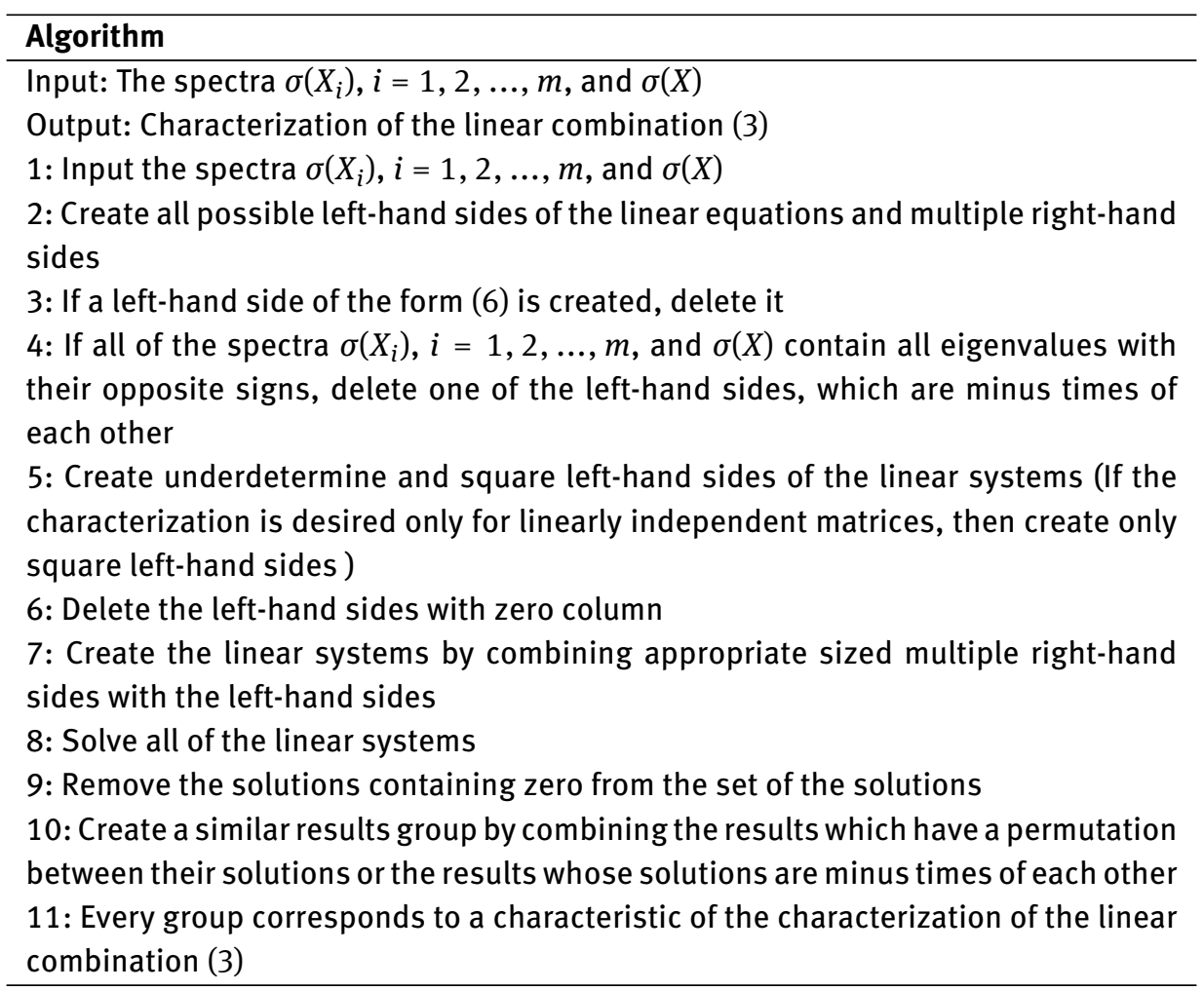

\subsection{Cubicity of linear combinations of two commuting cubic matrices}

The problem considered is to characterize situations in which a linear combination of the form

$$
c_{1} X_{1}+c_{2} X_{2}=X
$$

is a $\{\alpha, \beta, \gamma\}$-cubic matrix, where $X_{i} \in \mathbb{C}_{n}, i=1,2$, are matrices such that $\left(X_{i}-\alpha_{i} I_{n}\right)\left(X_{i}-\beta_{i} I_{n}\right)\left(X_{i}-\gamma_{i} I_{n}\right)=\mathbf{0}$ and $X_{1} X_{2}=X_{2} X_{1}$. It is well known that $\sigma\left(X_{i}\right) \subseteq\left\{\alpha_{i}, \beta_{i}, \gamma_{i}\right\}, i=1,2$. Accordingly, by the product rule there are $3 \cdot 3=9$ left-hand sides of linear equations, and the number of linear systems that need to be solved is $\left(\begin{array}{l}9 \\ 1\end{array}\right)+\left(\begin{array}{l}9 \\ 2\end{array}\right)=45$. 
Theorem 4. Let $X_{i} \in \mathbb{C}_{n}, i=1,2$, be nonzero commuting $\left\{\alpha_{i}, \beta_{i}, \gamma_{i}\right\}$-cubic matrices, and let $X$ be a linear combination of the form (7) with $c_{1}, c_{2} \in \mathbb{C} \backslash\{0\}$. Then $X$ is $a\{\alpha, \beta, \gamma\}$-cubic matrix if and only if there exists $a$ nonsingular matrix $S$ such that $c_{1} S^{-1} X_{1} S+c_{2} S^{-1} X_{2} S=S^{-1} X S$ is one of the following linear combinations:

$\left(a_{1}\right) c_{1}+\frac{\alpha_{2}}{\alpha_{1}} c_{2} \in\left\{\frac{\alpha}{\alpha_{1}}, \frac{\beta}{\alpha_{1}}, \frac{\gamma}{\alpha_{1}}\right\}, S^{-1} X_{1} S=\alpha_{1} I_{n}$ and $S^{-1} X_{2} S=\alpha_{2} I_{n}$,

$\left(a_{2}\right) c_{1}+\frac{\beta_{2}}{\alpha_{1}} c_{2} \in\left\{\frac{\alpha}{\alpha_{1}}, \frac{\beta}{\alpha_{1}}, \frac{\gamma}{\alpha_{1}}\right\}, S^{-1} X_{1} S=\alpha_{1} I_{n}$ and $S^{-1} X_{2} S=\beta_{2} I_{n}$,

$\left(a_{3}\right) c_{1}+\frac{\gamma_{2}}{\alpha_{1}} c_{2} \in\left\{\frac{\alpha}{\alpha_{1}}, \frac{\beta}{\alpha_{1}}, \frac{\gamma}{\alpha_{1}}\right\}, S^{-1} X_{1} S=\alpha_{1} I_{n}$ and $S^{-1} X_{2} S=\gamma_{2} I_{n}$,

$\left(a_{4}\right) c_{1}+\frac{\alpha_{2}}{\beta_{1}} c_{2} \in\left\{\frac{\alpha}{\alpha_{1}}, \frac{\beta}{\alpha_{1}}, \frac{\gamma}{\alpha_{1}}\right\}, S^{-1} X_{1} S=\beta_{1} I_{n}$ and $S^{-1} X_{2} S=\alpha_{2} I_{n}$,

$\left(a_{5}\right) c_{1}+\frac{\beta_{2}}{\beta_{1}} c_{2} \in\left\{\frac{\alpha}{\alpha_{1}}, \frac{\beta}{\alpha_{1}}, \frac{\gamma}{\alpha_{1}}\right\}, S^{-1} X_{1} S=\beta_{1} I_{n}$ and $S^{-1} X_{2} S=\beta_{2} I_{n}$,

$\left(a_{6}\right) c_{1}+\frac{\gamma_{2}}{\beta_{1}} c_{2} \in\left\{\frac{\alpha}{\alpha_{1}}, \frac{\beta}{\alpha_{1}}, \frac{\gamma}{\alpha_{1}}\right\}, S^{-1} X_{1} S=\beta_{1} I_{n}$ and $S^{-1} X_{2} S=\gamma_{2} I_{n}$,

$\left(a_{7}\right) c_{1}+\frac{\alpha_{2}}{\gamma_{1}} c_{2} \in\left\{\frac{\alpha}{\alpha_{1}}, \frac{\beta}{\alpha_{1}}, \frac{\gamma}{\alpha_{1}}\right\}, S^{-1} X_{1} S=\gamma_{1} I_{n}$ and $S^{-1} X_{2} S=\alpha_{2} I_{n}$,

$\left(a_{8}\right) c_{1}+\frac{\beta_{2}}{\gamma_{1}} c_{2} \in\left\{\frac{\alpha}{\alpha_{1}}, \frac{\beta}{\alpha_{1}}, \frac{\gamma}{\alpha_{1}}\right\}, S^{-1} X_{1} S=\gamma_{1} I_{n}$ and $S^{-1} X_{2} S=\beta_{2} I_{n}$,

$\left(a_{9}\right) c_{1}+\frac{\gamma_{2}}{\gamma_{1}} c_{2} \in\left\{\frac{\alpha}{\alpha_{1}}, \frac{\beta}{\alpha_{1}}, \frac{\gamma}{\alpha_{1}}\right\}, S^{-1} X_{1} S=\gamma_{1} I_{n}$ and $S^{-1} X_{2} S=\gamma_{2} I_{n}$,

$\left(a_{10}\right)\left(c_{1}, c_{2}\right) \in\left\{\begin{array}{l}\left(\frac{-\alpha \beta_{2}+\alpha_{2} \beta}{\alpha_{1}\left(\alpha_{2}-\beta_{2}\right)}, \frac{\alpha-\beta}{\alpha_{2}-\beta_{2}}\right),\left(\frac{-\alpha \beta_{2}+\alpha_{2} \gamma}{\alpha_{1}\left(\alpha_{2}-\beta_{2}\right)}, \frac{\alpha-\gamma}{\alpha_{2}-\beta_{2}}\right),\left(\frac{\alpha_{2} \alpha-\beta_{2} \beta}{\alpha_{1}\left(\alpha_{2}-\beta_{2}\right)}, \frac{\beta-\alpha}{\alpha_{2}-\beta_{2}}\right), \\ \left.\frac{-\beta_{2} \beta+\alpha_{2} \gamma}{\alpha_{1}\left(\alpha_{2}-\beta_{2}\right)}, \frac{\beta-\gamma}{\alpha_{2}-\beta_{2}}\right),\left(\frac{\alpha_{2} \alpha-\beta_{2} \gamma}{\alpha_{1}\left(\alpha_{2}-\beta_{2}\right)}, \frac{\gamma-\alpha}{\alpha_{2}-\beta_{2}}\right),\left(\frac{\alpha_{2} \beta-\beta_{2} \gamma}{\alpha_{1}\left(\alpha_{2}-\beta_{2}\right)}, \frac{\gamma-\beta}{\alpha_{2}-\beta_{2}}\right)\end{array}\right\}, S^{-1} X_{1} S=\alpha_{1} I_{n_{1}} \oplus \alpha_{1} I_{n_{2}}$ and $S^{-1} X_{2} S=\alpha_{2} I_{n_{1}} \oplus \beta_{2} I_{n_{2}}$,

$\left(a_{11}\right)\left(c_{1}, c_{2}\right) \in\left\{\begin{array}{ll}\left(\frac{-\alpha \gamma_{2}+\alpha_{2} \beta}{\alpha_{1}\left(\alpha_{2}-\gamma_{2}\right)}, \frac{\alpha-\beta}{\alpha_{2}-\gamma_{2}}\right),\left(\frac{-\alpha \gamma_{2}+\alpha_{2} \gamma}{\alpha_{1}\left(\alpha_{2}-\gamma_{2}\right)}, \frac{\alpha-\gamma}{\alpha_{2}-\gamma_{2}}\right),\left(\frac{\alpha_{2} \alpha-\gamma_{2} \beta}{\alpha_{1}\left(\alpha_{2}-\gamma_{2}\right)}, \frac{\beta-\alpha}{\alpha_{2}-\gamma_{2}}\right), \\ \left(\frac{-\gamma_{2} \beta+\alpha_{2} \gamma}{\alpha_{1}\left(\alpha_{2}-\gamma_{2}\right)}, \frac{\beta-\gamma}{\alpha_{2}-\gamma_{2}}\right),\left(\frac{\alpha_{2} \alpha-\gamma_{2} \gamma}{\alpha_{1}\left(\alpha_{2}-\gamma_{2}\right)}, \frac{\gamma-\alpha}{\alpha_{2}-\gamma_{2}}\right),\left(\frac{\alpha_{2} \beta-\gamma_{2} \gamma}{\alpha_{1}\left(\alpha_{2}-\gamma_{2}\right)}, \frac{\gamma-\beta}{\alpha_{2}-\gamma_{2}}\right)\end{array}\right\}, S^{-1} X_{1} S=\alpha_{1} I_{n_{1}} \oplus \alpha_{1} I_{n_{2}}$ and $S^{-1} X_{2} S=\alpha_{2} I_{n_{1}} \oplus \gamma_{2} I_{n_{2}}$,

$\left(a_{12}\right)\left(c_{1}, c_{2}\right) \in\left\{\begin{array}{l}\left(\frac{\alpha-\gamma}{\alpha_{1}-\beta_{1}}, \frac{-\alpha \beta_{1}+\alpha_{1} \beta}{\alpha_{2}\left(\alpha_{1}-\beta_{1}\right)}\right),\left(\frac{\alpha-\beta}{\alpha_{1}-\beta_{1}}, \frac{-\alpha \beta_{1}+\alpha_{1} \gamma}{\alpha_{2}\left(\alpha_{1}-\beta_{1}\right)}\right),\left(\frac{\beta-\alpha}{\alpha_{1}-\beta_{1}}, \frac{\alpha_{1} \alpha-\beta_{1} \beta}{\alpha_{2}\left(\alpha_{1}-\beta_{1}\right)}\right), \\ \left.\frac{\beta-\gamma}{\alpha_{1}-\beta_{1}}, \frac{-\beta_{1} \beta+\alpha_{1} \gamma}{\alpha_{2}\left(\alpha_{1}-\beta_{1}\right)}\right),\left(\frac{\gamma-\alpha}{\alpha_{1}-\beta_{1}}, \frac{\alpha_{1} \alpha-\beta_{1} \gamma}{\alpha_{2}\left(\alpha_{1}-\beta_{1}\right)}\right),\left(\frac{\gamma-\beta}{\alpha_{1}-\beta_{1}}, \frac{\alpha_{1} \beta-\beta_{1} \gamma}{\alpha_{2}\left(\alpha_{1}-\beta_{1}\right)}\right)\end{array}\right\}, S^{-1} X_{1} S=\alpha_{1} I_{n_{1}} \oplus \beta_{1} I_{n_{2}}$ and $S^{-1} X_{2} S=\alpha_{2} I_{n_{1}} \oplus \alpha_{2} I_{n_{2}}$,

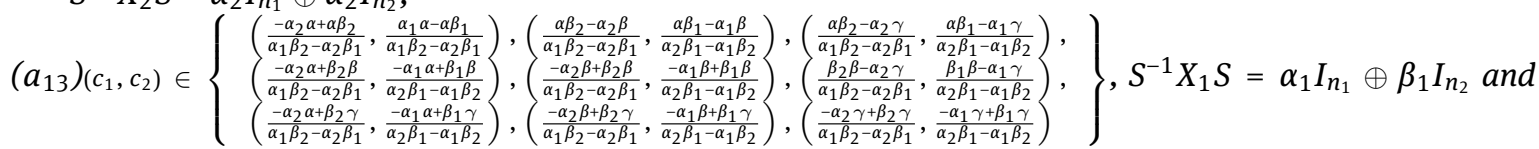
$S^{-1} X_{2} S=\alpha_{2} I_{n_{1}} \oplus \beta_{2} I_{n_{2}}$,

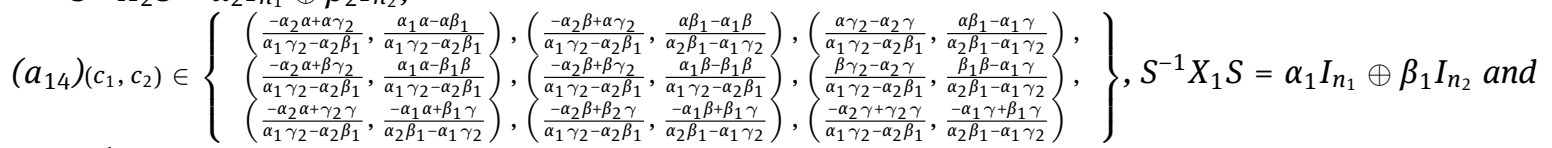
$S^{-1} X_{2} S=\alpha_{2} I_{n_{1}} \oplus \gamma_{2} I_{n_{2}}$,

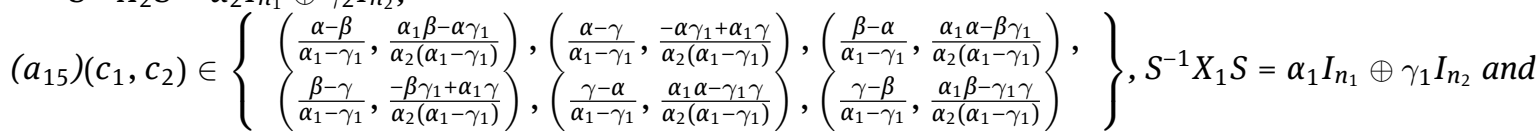
$S^{-1} X_{2} S=\alpha_{2} I_{n_{1}} \oplus \alpha_{2} I_{n_{2}}$,

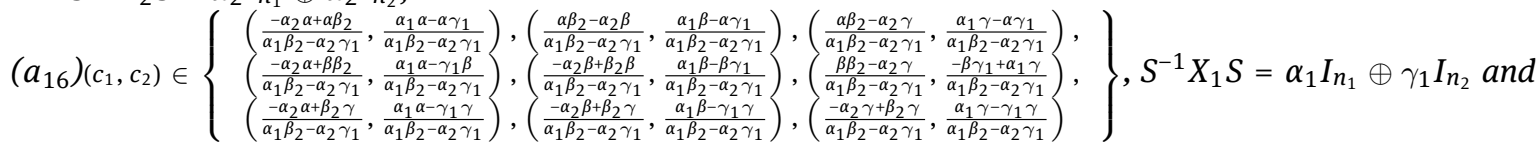
$S^{-1} X_{2} S=\alpha_{2} I_{n_{1}} \oplus \beta_{2} I_{n_{2}}$,

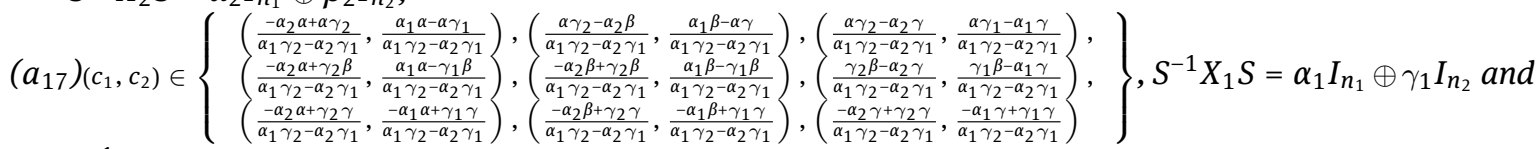
$S^{-1} X_{2} S=\alpha_{2} I_{n_{1}} \oplus \gamma_{2} I_{n_{2}}$,

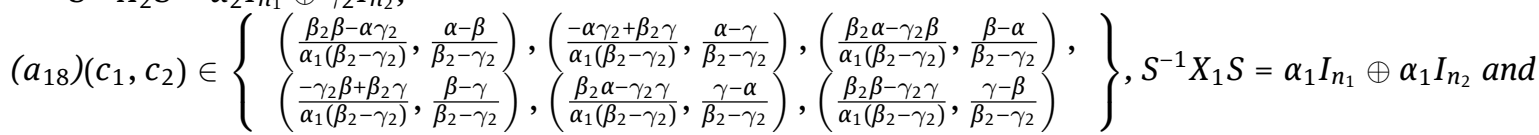
$S^{-1} X_{2} S=\beta_{2} I_{n_{1}} \oplus \gamma_{2} I_{n_{2}}$, 


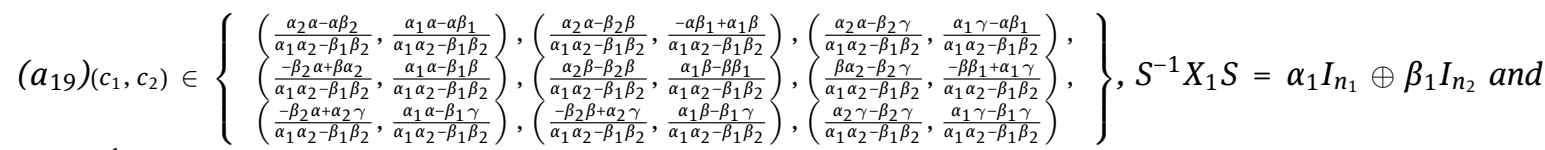
$S^{-1} X_{2} S=\beta_{2} I_{n_{1}} \oplus \alpha_{2} I_{n_{2}}$,

$\left(a_{20}\right)\left(c_{1}, c_{2}\right) \in\left\{\begin{array}{l}\left(\frac{\alpha-\beta}{\alpha_{1}-\beta_{1}}, \frac{\alpha_{1} \beta-\alpha \beta_{1}}{\beta_{2}\left(\alpha_{1}-\beta_{1}\right)}\right),\left(\frac{\alpha-\gamma}{\alpha_{1}-\beta_{1}}, \frac{-\alpha \beta_{1}+\alpha_{1} \gamma}{\beta_{2}\left(\alpha_{1}-\beta_{1}\right)}\right),\left(\frac{\beta-\alpha}{\alpha_{1}-\beta_{1}}, \frac{\alpha_{1} \alpha-\beta \beta_{1}}{\beta_{2}\left(\alpha_{1}-\beta_{1}\right)}\right), \\ \left(\frac{\beta-\gamma}{\alpha_{1}-\beta_{1}}, \frac{-\beta \beta_{1}+\alpha_{1} \gamma}{\beta_{2}\left(\alpha_{1}-\beta_{1}\right)}\right),\left(\frac{\gamma-\alpha}{\alpha_{1}-\beta_{1}}, \frac{\alpha_{1} \alpha-\beta_{1} \gamma}{\beta_{2}\left(\alpha_{1}-\beta_{1}\right)}\right),\left(\frac{\gamma-\beta}{\alpha_{1}-\beta_{1}}, \frac{\alpha_{1} \beta-\beta_{1} \gamma}{\beta_{2}\left(\alpha_{1}-\beta_{1}\right)}\right)\end{array}\right\}, S^{-1} X_{1} S=\alpha_{1} I_{n_{1}} \oplus \beta_{1} I_{n_{2}}$ and $S^{-1} X_{2} S=\beta_{2} I_{n_{1}} \oplus \beta_{2} I_{n_{2}}$,

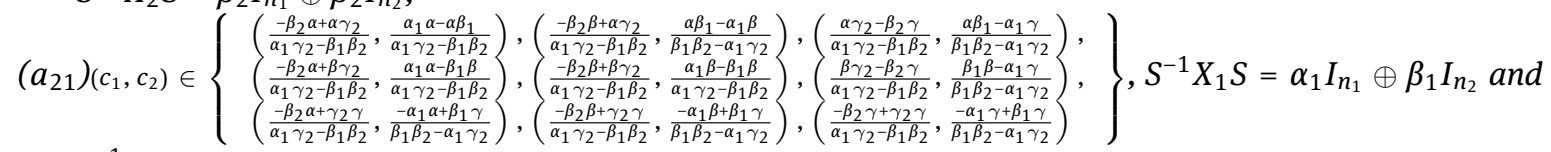
$S^{-1} X_{2} S=\beta_{2} I_{n_{1}} \oplus \gamma_{2} I_{n_{2}}$,

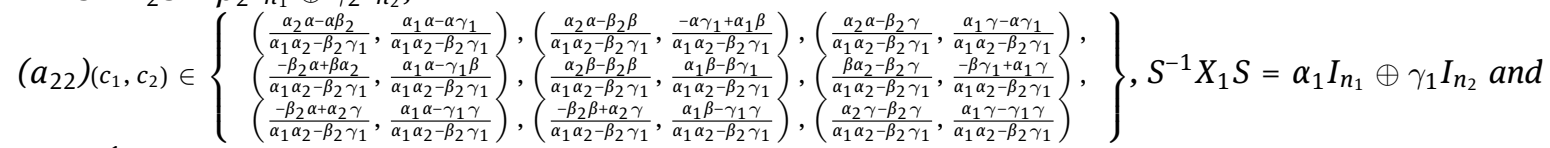
$S^{-1} X_{2} S=\beta_{2} I_{n_{1}} \oplus \gamma_{2} I_{n_{2}}$,

$\left(a_{23}\right)\left(c_{1}, c_{2}\right) \in\left\{\begin{array}{l}\left(\frac{\alpha-\beta}{\alpha_{1}-\gamma_{1}}, \frac{\alpha_{1} \beta-\alpha \gamma_{1}}{\beta_{2}\left(\alpha_{1}-\gamma_{1}\right)}\right),\left(\frac{\alpha-\gamma}{\alpha_{1}-\gamma_{1}}, \frac{-\alpha \gamma_{1}+\alpha_{1} \gamma}{\beta_{2}\left(\alpha_{1}-\gamma_{1}\right)}\right),\left(\frac{\beta-\alpha}{\alpha_{1}-\gamma_{1}}, \frac{\alpha_{1} \alpha-\beta \gamma_{1}}{\beta_{2}\left(\alpha_{1}-\gamma_{1}\right)}\right), \\ \left.\frac{\beta-\gamma}{\alpha_{1}-\gamma_{1}}, \frac{-\beta \gamma_{1}+\alpha_{1} \gamma}{\beta_{2}\left(\alpha_{1}-\gamma_{1}\right)}\right),\left(\frac{\gamma-\alpha}{\alpha_{1}-\gamma_{1}}, \frac{\alpha_{1} \alpha-\gamma_{1} \gamma}{\beta_{2}\left(\alpha_{1}-\gamma_{1}\right)}\right),\left(\frac{\gamma-\beta}{\alpha_{1}-\gamma_{1}}, \frac{\alpha_{1} \beta-\gamma_{1} \gamma}{\beta_{2}\left(\alpha_{1}-\gamma_{1}\right)}\right)\end{array}\right\}, S^{-1} X_{1} S=\alpha_{1} I_{n_{1}} \oplus \gamma_{1} I_{n_{2}}$ and $S^{-1} X_{2} S=\beta_{2} I_{n_{1}} \oplus \beta_{2} I_{n_{2}}$,

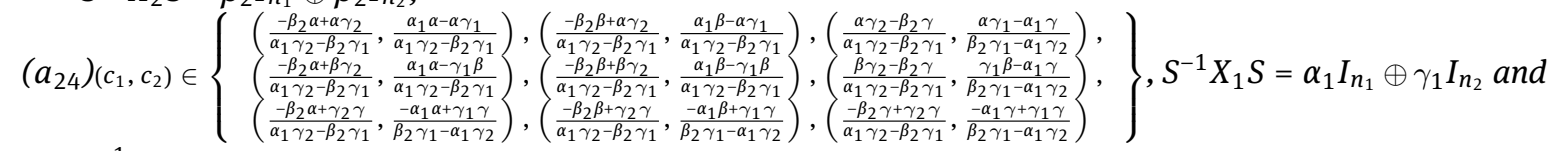
$S^{-1} X_{2} S=\beta_{2} I_{n_{1}} \oplus \gamma_{2} I_{n_{2}}$,

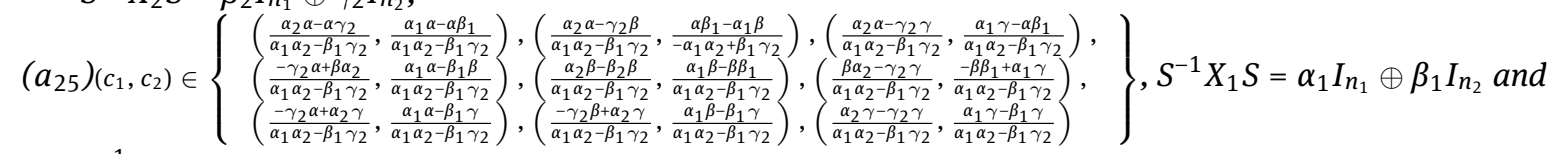
$S^{-1} X_{2} S=\gamma_{2} I_{n_{1}} \oplus \alpha_{2} I_{n_{2}}$,

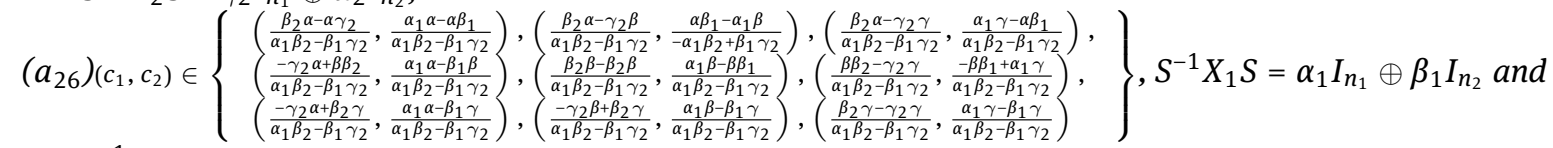
$S^{-1} X_{2} S=\gamma_{2} I_{n_{1}} \oplus \beta_{2} I_{n_{2}}$,

$\left(a_{27}\right)\left(c_{1}, c_{2}\right) \in\left\{\begin{array}{l}\left(\frac{\alpha-\beta}{\alpha_{1}-\beta_{1}}, \frac{\alpha \beta_{1}-\alpha_{1} \beta}{\gamma_{2}\left(-\alpha_{1}+\beta_{1}\right)}\right),\left(\frac{\alpha-\gamma}{\alpha_{1}-\beta_{1}}, \frac{-\alpha \beta_{1}+\alpha_{1} \gamma}{\gamma_{2}\left(\alpha_{1}-\beta_{1}\right)}\right),\left(\frac{\beta-\alpha}{\alpha_{1}-\beta_{1}}, \frac{\alpha_{1} \alpha-\beta \beta_{1}}{\gamma_{2}\left(\alpha_{1}-\beta_{1}\right)}\right), \\ \left(\frac{\beta-\gamma}{\alpha_{1}-\beta_{1}}, \frac{-\beta \beta_{1}+\alpha_{1} \gamma}{\gamma_{2}\left(\alpha_{1}-\beta_{1}\right)}\right),\left(\frac{\gamma-\alpha}{\alpha_{1}-\beta_{1}}, \frac{\alpha_{1} \alpha-\beta_{1} \gamma}{\gamma_{2}\left(\alpha_{1}-\beta_{1}\right)}\right),\left(\frac{\gamma-\beta}{\alpha_{1}-\beta_{1}}, \frac{\alpha_{1} \beta-\beta_{1} \gamma}{\gamma_{2}\left(\alpha_{1}-\beta_{1}\right)}\right)\end{array}\right\}, S^{-1} X_{1} S=\alpha_{1} I_{n_{1}} \oplus \beta_{1} I_{n_{2}}$ and $S^{-1} X_{2} S=\gamma_{2} I_{n_{1}} \oplus \gamma_{2} I_{n_{2}}$,

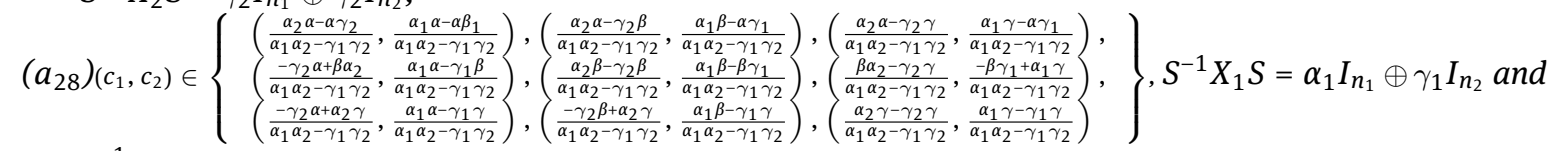
$S^{-1} X_{2} S=\gamma_{2} I_{n_{1}} \oplus \alpha_{2} I_{n_{2}}$,

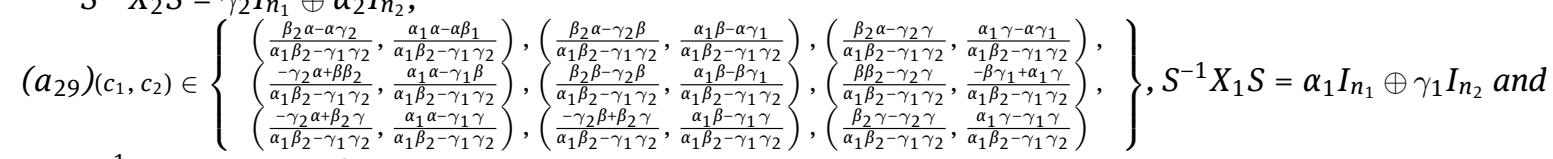
$S^{-1} X_{2} S=\gamma_{2} I_{n_{1}} \oplus \beta_{2} I_{n_{2}}$,

$\left(a_{30}\right)\left(c_{1}, c_{2}\right) \in\left\{\begin{array}{l}\left(\frac{\alpha-\beta}{\alpha_{1}-\gamma_{1}}, \frac{\alpha \gamma_{1}-\alpha_{1} \beta}{\gamma_{2}\left(-\alpha_{1}+\gamma_{1}\right)}\right),\left(\frac{\alpha-\gamma}{\alpha_{1}-\gamma_{1}}, \frac{-\alpha \gamma_{1}+\alpha_{1} \gamma}{\gamma_{2}\left(\alpha_{1}-\gamma_{1}\right)}\right),\left(\frac{\beta-\alpha}{\alpha_{1}-\gamma_{1}}, \frac{\alpha_{1} \alpha-\beta \gamma_{1}}{\gamma_{2}\left(\alpha_{1}-\gamma_{1}\right)}\right), \\ \left(\frac{\beta-\gamma}{\alpha_{1}-\gamma_{1}}, \frac{-\beta \gamma_{1}+\alpha_{1} \gamma}{\gamma_{2}\left(\alpha_{1}-\gamma_{1}\right)}\right),\left(\frac{\gamma-\alpha}{\alpha_{1}-\gamma_{1}}, \frac{\alpha_{1} \alpha-\gamma_{1} \gamma}{\gamma_{2}\left(\alpha_{1}-\gamma_{1}\right)}\right),\left(\frac{\gamma-\beta}{\alpha_{1}-\gamma_{1}}, \frac{\alpha_{1} \beta-\gamma_{1} \gamma}{\gamma_{2}\left(\alpha_{1}-\gamma_{1}\right)}\right)\end{array}\right\}, S^{-1} X_{1} S=\alpha_{1} I_{n_{1}} \oplus \gamma_{1} I_{n_{2}}$ and $S^{-1} X_{2} S=\gamma_{2} I_{n_{1}} \oplus \gamma_{2} I_{n_{2}}$,

$\left(a_{31}\right)\left(c_{1}, c_{2}\right) \in\left\{\begin{array}{l}\left(\frac{-\alpha \beta_{2}+\alpha_{2} \beta}{\beta_{1}\left(\alpha_{2}-\beta_{2}\right)}, \frac{\alpha-\beta}{\alpha_{2}-\beta_{2}}\right),\left(\frac{-\alpha \beta_{2}+\alpha_{2} \gamma}{\beta_{1}\left(\alpha_{2}-\beta_{2}\right)}, \frac{\alpha-\gamma}{\alpha_{2}-\beta_{2}}\right),\left(\frac{\alpha_{2} \alpha-\beta_{2} \beta}{\beta_{1}\left(\alpha_{2}-\beta_{2}\right)}, \frac{\beta-\alpha}{\alpha_{2}-\beta_{2}}\right), \\ \left.\frac{-\beta_{2} \beta+\alpha_{2} \gamma}{\beta_{1}\left(\alpha_{2}-\beta_{2}\right)}, \frac{\beta-\gamma}{\alpha_{2}-\beta_{2}}\right),\left(\frac{\alpha_{2} \alpha-\beta_{2} \gamma}{\beta_{1}\left(\alpha_{2}-\beta_{2}\right)}, \frac{\gamma-\alpha}{\alpha_{2}-\beta_{2}}\right),\left(\frac{\alpha_{2} \beta-\beta_{2} \gamma}{\beta_{1}\left(\alpha_{2}-\beta_{2}\right)}, \frac{\gamma-\beta}{\alpha_{2}-\beta_{2}}\right)\end{array}\right\}, S^{-1} X_{1} S=\beta_{1} I_{n_{1}} \oplus \beta_{1} I_{n_{2}}$ and $S^{-1} X_{2} S=\alpha_{2} I_{n_{1}} \oplus \beta_{2} I_{n_{2}}$, 


$$
\begin{aligned}
& \left(a_{32}\right)\left(c_{1}, c_{2}\right) \in\left\{\begin{array}{l}
\left(\frac{-\alpha \gamma_{2}+\alpha_{2} \beta}{\beta_{1}\left(\alpha_{2}-\gamma_{2}\right)}, \frac{\alpha-\beta}{\alpha_{2}-\gamma_{2}}\right),\left(\frac{-\alpha \gamma_{2}+\alpha_{2} \gamma}{\beta_{1}\left(\alpha_{2}-\gamma_{2}\right)}, \frac{\alpha-\gamma}{\alpha_{2}-\gamma_{2}}\right),\left(\frac{\alpha_{2} \alpha-\gamma_{2} \beta}{\beta_{1}\left(\alpha_{2}-\gamma_{2}\right)}, \frac{\beta-\alpha}{\alpha_{2}-\gamma_{2}}\right), \\
\left(\frac{-\gamma_{2} \beta+\alpha_{2} \gamma}{\beta_{1}\left(\alpha_{2}-\gamma_{2}\right)}, \frac{\beta-\gamma}{\alpha_{2}-\gamma_{2}}\right),\left(\frac{\alpha_{2} \alpha-\gamma_{2} \gamma}{\beta_{1}\left(\alpha_{2}-\gamma_{2}\right)}, \frac{\gamma-\alpha}{\alpha_{2}-\gamma_{2}}\right),\left(\frac{\alpha_{2} \beta-\gamma_{2} \gamma}{\beta_{1}\left(\alpha_{2}-\gamma_{2}\right)}, \frac{\gamma-\beta}{\alpha_{2}-\gamma_{2}}\right)
\end{array}\right\}, S^{-1} X_{1} S=\beta_{1} I_{n_{1}} \oplus \beta_{1} I_{n_{2}} \text { and } \\
& S^{-1} X_{2} S=\alpha_{2} I_{n_{1}} \oplus \gamma_{2} I_{n_{2}} \text {, } \\
& \left(a_{33}\right)\left(c_{1}, c_{2}\right) \in\left\{\begin{array}{l}
\left(\frac{\alpha-\beta}{\beta_{1}-\gamma_{1}}, \frac{\alpha \gamma_{1}-\beta_{1} \beta}{\alpha_{2}\left(-\beta_{1}+\gamma_{1}\right)}\right),\left(\frac{\alpha-\gamma}{\beta_{1}-\gamma_{1}}, \frac{\alpha \gamma_{1}+\beta_{1} \gamma}{\alpha_{2}\left(\beta_{1}-\gamma_{1}\right)}\right),\left(\frac{\beta-\alpha}{\beta_{1}-\gamma_{1}}, \frac{\beta_{1} \alpha-\beta \gamma_{1}}{\alpha_{2}\left(\beta_{1}-\gamma_{1}\right)}\right), \\
\left(\frac{\beta-\gamma}{\beta_{1}-\gamma_{1}}, \frac{-\beta \gamma_{1}+\beta_{1} \gamma}{\alpha_{2}\left(\beta_{1}-\gamma_{1}\right)}\right),\left(\frac{\gamma-\alpha}{\beta_{1}-\gamma_{1}}, \frac{\beta_{1} \alpha-\gamma_{1} \gamma}{\alpha_{2}\left(\beta_{1}-\gamma_{1}\right)}\right),\left(\frac{\gamma-\beta}{\beta_{1}-\gamma_{1}}, \frac{\beta_{1} \beta-\gamma_{1} \gamma}{\alpha_{2}\left(\beta_{1}-\gamma_{1}\right)}\right)
\end{array}\right\}, S^{-1} X_{1} S=\beta_{1} I_{n_{1}} \oplus \gamma_{1} I_{n_{2}} \text { and } \\
& S^{-1} X_{2} S=\alpha_{2} I_{n_{1}} \oplus \alpha_{2} I_{n_{2}} \text {, }
\end{aligned}
$$

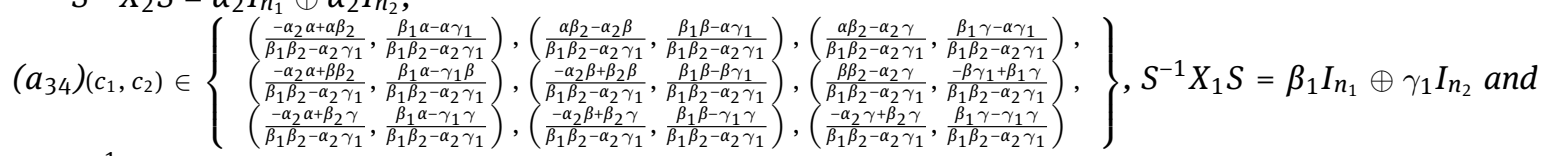

$$
\begin{aligned}
& S^{-1} X_{2} S=\alpha_{2} I_{n_{1}} \oplus \beta_{2} I_{n_{2}} \text {, }
\end{aligned}
$$

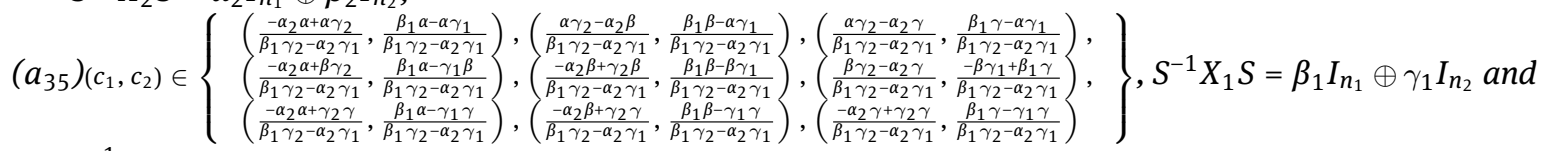

$$
\begin{aligned}
& S^{-1} X_{2} S=\alpha_{2} I_{n_{1}} \oplus \gamma_{2} I_{n_{2}} \text {, }
\end{aligned}
$$

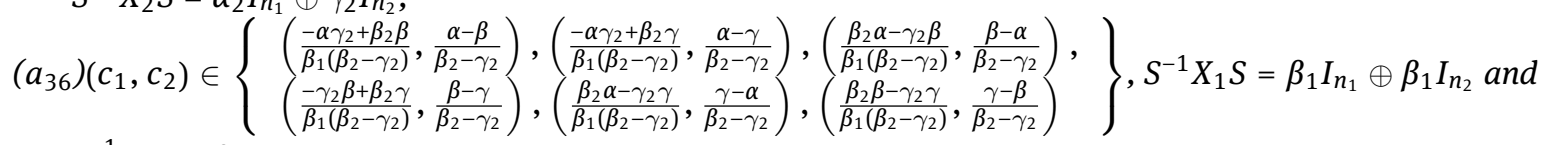

$$
\begin{aligned}
& S^{-1} X_{2} S=\beta_{2} I_{n_{1}} \oplus \gamma_{2} I_{n_{2}} \text {, }
\end{aligned}
$$

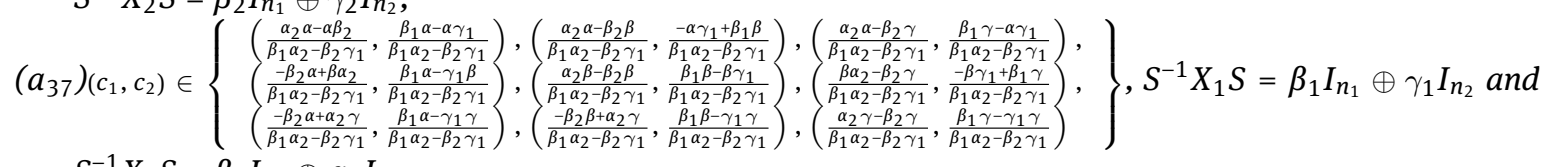
$S^{-1} X_{2} S=\beta_{2} I_{n_{1}} \oplus \alpha_{2} I_{n_{2}}$,

$\left(a_{38}\right)\left(c_{1}, c_{2}\right) \in\left\{\begin{array}{l}\left(\frac{\alpha-\beta}{\beta_{1}-\gamma_{1}}, \frac{\beta_{1} \beta-\alpha \gamma_{1}}{\beta_{2}\left(\beta_{1}-\gamma_{1}\right)}\right),\left(\frac{\alpha-\gamma}{\beta_{1}-\gamma_{1}}, \frac{-\alpha \gamma_{1}+\beta_{1} \gamma}{\beta_{2}\left(\beta_{1}-\gamma_{1}\right)}\right),\left(\frac{\beta-\alpha}{\beta_{1}-\gamma_{1}}, \frac{\beta_{1} \alpha-\beta \gamma_{1}}{\beta_{2}\left(\beta_{1}-\gamma_{1}\right)}\right), \\ \left.\frac{\beta-\gamma}{\beta_{1}-\gamma_{1}}, \frac{-\beta \gamma_{1}+\beta_{1} \gamma}{\beta_{2}\left(\beta_{1}-\gamma_{1}\right)}\right),\left(\frac{\gamma-\alpha}{\beta_{1}-\gamma_{1}}, \frac{\beta_{1} \alpha-\gamma_{1} \gamma}{\beta_{2}\left(\beta_{1}-\gamma_{1}\right)}\right),\left(\frac{\gamma-\beta}{\beta_{1}-\gamma_{1}}, \frac{\beta_{1} \beta-\gamma_{1} \gamma}{\beta_{2}\left(\beta_{1}-\gamma_{1}\right)}\right.\end{array}\right), S^{-1} X_{1} S=\beta_{1} I_{n_{1}} \oplus \gamma_{1} I_{n_{2}}$ and $S^{-1} X_{2} S=\beta_{2} I_{n_{1}} \oplus \beta_{2} I_{n_{2}}$,

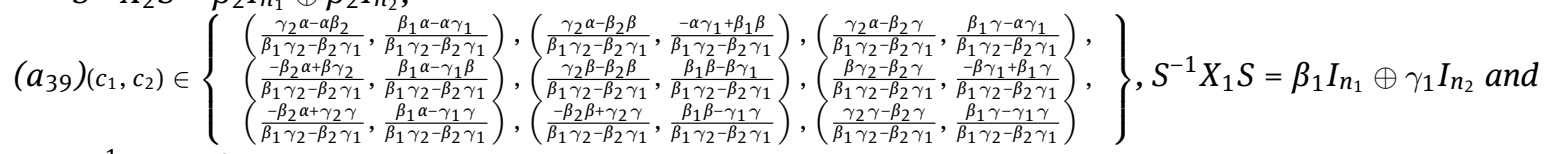
$S^{-1} X_{2} S=\beta_{2} I_{n_{1}} \oplus \gamma_{2} I_{n_{2}}$,

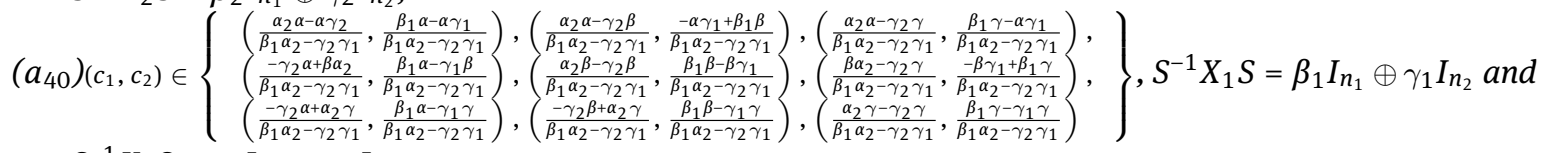
$S^{-1} X_{2} S=\gamma_{2} I_{n_{1}} \oplus \alpha_{2} I_{n_{2}}$

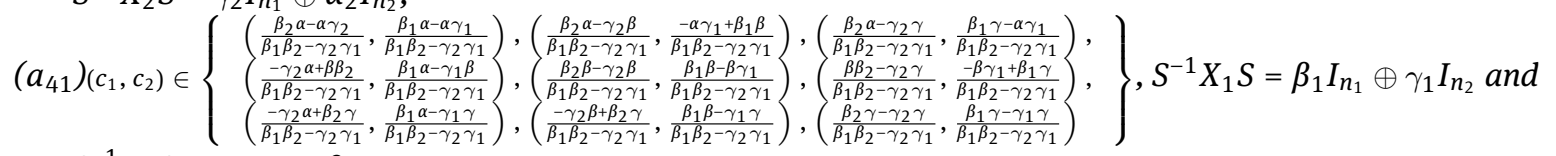
$S^{-1} X_{2} S=\gamma_{2} I_{n_{1}} \oplus \beta_{2} I_{n_{2}}$,

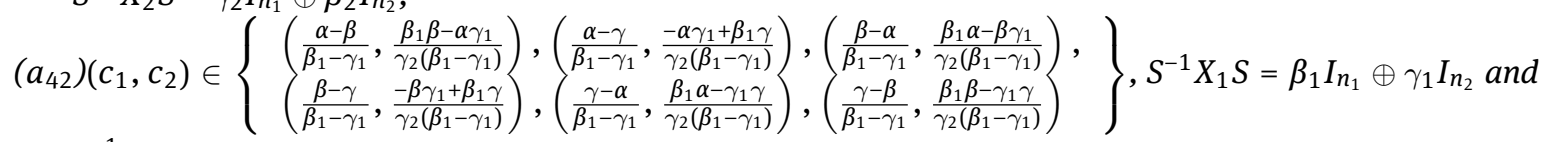
$S^{-1} X_{2} S=\gamma_{2} I_{n_{1}} \oplus \gamma_{2} I_{n_{2}}$,

$\left(a_{43}\right)\left(c_{1}, c_{2}\right) \in\left\{\begin{array}{l}\left(\frac{-\alpha \beta_{2}+\alpha_{2} \beta}{\gamma_{1}\left(\alpha_{2}-\beta_{2}\right)}, \frac{\alpha-\beta}{\alpha_{2}-\beta_{2}}\right),\left(\frac{-\alpha \beta_{2}+\alpha_{2} \gamma}{\gamma_{1}\left(\alpha_{2}-\beta_{2}\right)}, \frac{\alpha-\gamma}{\alpha_{2}-\beta_{2}}\right),\left(\frac{\alpha_{2} \alpha-\beta_{2} \beta}{\gamma_{1}\left(\alpha_{2}-\beta_{2}\right)}, \frac{\beta-\alpha}{\alpha_{2}-\beta_{2}}\right), \\ \left(\frac{-\beta_{2} \beta+\alpha_{2} \gamma}{\gamma_{1}\left(\alpha_{2}-\beta_{2}\right)}, \frac{\beta-\gamma}{\alpha_{2}-\beta_{2}}\right),\left(\frac{\alpha_{2} \alpha-\beta_{2} \gamma}{\gamma_{1}\left(\alpha_{2}-\beta_{2}\right)}, \frac{\gamma-\alpha}{\alpha_{2}-\beta_{2}}\right),\left(\frac{\alpha_{2} \beta-\beta_{2} \gamma}{\gamma_{1}\left(\alpha_{2}-\beta_{2}\right)}, \frac{\gamma-\beta}{\alpha_{2}-\beta_{2}}\right)\end{array}\right\}, S^{-1} X_{1} S=\gamma_{1} I_{n_{1}} \oplus \gamma_{1} I_{n_{2}}$ and $S^{-1} X_{2} S=\alpha_{2} I_{n_{1}} \oplus \beta_{2} I_{n_{2}}$,

$\left(a_{44}\right)\left(c_{1}, c_{2}\right) \in\left\{\begin{array}{l}\left(\frac{-\alpha \gamma_{2}+\alpha_{2} \beta}{\gamma_{1}\left(\alpha_{2}-\gamma_{2}\right)}, \frac{\alpha-\beta}{\alpha_{2}-\gamma_{2}}\right),\left(\frac{-\alpha \gamma_{2}+\alpha_{2} \gamma}{\gamma_{1}\left(\alpha_{2}-\gamma_{2}\right)}, \frac{\alpha-\gamma}{\alpha_{2}-\gamma_{2}}\right),\left(\frac{\alpha_{2} \alpha-\gamma_{2} \beta}{\gamma_{1}\left(\alpha_{2}-\gamma_{2}\right)}, \frac{\beta-\alpha}{\alpha_{2}-\gamma_{2}}\right), \\ \left(\frac{-\gamma_{2} \beta+\alpha_{2} \gamma}{\gamma_{1}\left(\alpha_{2}-\gamma_{2}\right)}, \frac{\beta-\gamma}{\alpha_{2}-\gamma_{2}}\right),\left(\frac{\alpha_{2} \alpha-\gamma_{2} \gamma}{\gamma_{1}\left(\alpha_{2}-\gamma_{2}\right)}, \frac{\gamma-\alpha}{\alpha_{2}-\gamma_{2}}\right),\left(\frac{\alpha_{2} \beta-\gamma_{2} \gamma}{\gamma_{1}\left(\alpha_{2}-\gamma_{2}\right)}, \frac{\gamma-\beta}{\alpha_{2}-\gamma_{2}}\right)\end{array}\right\}, S^{-1} X_{1} S=\gamma_{1} I_{n_{1}} \oplus \gamma_{1} I_{n_{2}}$ and $S^{-1} X_{2} S=\alpha_{2} I_{n_{1}} \oplus \gamma_{2} I_{n_{2}}$, 


$$
\begin{gathered}
\left(a_{45}\right)\left(c_{1}, c_{2}\right) \in\left\{\begin{array}{l}
\left(\frac{-\alpha \gamma_{2}+\beta_{2} \beta}{\gamma_{1}\left(\beta_{2}-\gamma_{2}\right)}, \frac{\alpha-\beta}{\beta_{2}-\gamma_{2}}\right),\left(\frac{-\alpha \gamma_{2}+\beta_{2} \gamma}{\gamma_{1}\left(\beta_{2}-\gamma_{2}\right)}, \frac{\alpha-\gamma}{\beta_{2}-\gamma_{2}}\right),\left(\frac{\beta_{2} \alpha-\gamma_{2} \beta}{\gamma_{1}\left(\beta_{2}-\gamma_{2}\right)}, \frac{\beta-\alpha}{\beta_{2}-\gamma_{2}}\right), \\
\left(\frac{-\gamma_{2} \beta+\beta_{2} \gamma}{\gamma_{1}\left(\beta_{2}-\gamma_{2}\right)}, \frac{\beta-\gamma}{\beta_{2}-\gamma_{2}}\right),\left(\frac{\beta_{2} \alpha-\gamma_{2} \gamma}{\gamma_{1}\left(\beta_{2}-\gamma_{2}\right)}, \frac{\gamma-\alpha}{\beta_{2}-\gamma_{2}}\right),\left(\frac{\beta_{2} \beta-\gamma_{2} \gamma}{\gamma_{1}\left(\beta_{2}-\gamma_{2}\right)}, \frac{\gamma-\beta}{\beta_{2}-\gamma_{2}}\right)
\end{array}\right\}, S^{-1} X_{1} S=\gamma_{1} I_{n_{1}} \oplus \gamma_{1} I_{n_{2}} \text { and } \\
S^{-1} X_{2} S=\beta_{2} I_{n_{1}} \oplus \gamma_{2} I_{n_{2}} .
\end{gathered}
$$

These results are a generalization of the results given for tripotent matrices in [5] and an expanding of the results given for quadratic matrices given in [27].

\subsection{Quadripotency of linear combinations of two commuting quadripotent matrices}

The problem considered is to characterize all situations in which a linear combination of the form

$$
c_{1} Q_{1}+c_{2} Q_{2}=Q
$$

is a quadripotent matrix, when $Q_{i} \in \mathbb{C}_{n}, i=1,2$, are both nonzero quadripotent matrices such that $Q_{1} Q_{2}=$ $Q_{2} Q_{1}$. The spectra of the quadripotent matrices are subsets of the set $\left\{-\frac{1}{2}+\frac{\sqrt{3} i}{2},-\frac{1}{2}-\frac{\sqrt{3} i}{2}, 1,0\right\}[19$, Theorem $1]$, and thus by the product rule there are $4 \cdot 4=16$ left-hand sides of the linear equations. However, since one of these 16 left-hand sides is $0 c_{1}+0 c_{2}$, there are 15 left-hand sides that need to be considered. Hence, the number of linear systems that need to be solved is $\left(\begin{array}{c}15 \\ 1\end{array}\right)+\left(\begin{array}{c}15 \\ 2\end{array}\right)=120$. Moreover, direct calculations show that the matrix $Q$ of the form (8) is quadripotent if and only if

$$
\left(c_{1}^{4}-c_{1}\right) Q_{1}+4 c_{1}^{3} c_{2} Q_{1}^{3} Q_{2}+6 c_{1}^{2} c_{2}^{2} Q_{1}^{2} Q_{2}^{2}+4 c_{1} c_{2}^{3} Q_{1} Q_{2}^{3}+\left(c_{2}^{4}-c_{2}\right) Q_{2}=\mathbf{0} .
$$

The equation (9) is utilized in the process of determining the conditions on the matrices in the linear systems whose set of solutions consists of unique solutions.

Theorem 5. Let $Q_{1}, Q_{2} \in \mathbb{C}_{n}$ be nonzero commuting quadripotent matrices, and let $Q$ be a linear combination of the form (8) with $c_{1}, c_{2} \in \mathbb{C} \backslash\{0\}$. Then, $Q$ is quadripotent if and only if:

$\left(b_{1}\right) c_{1}+c_{2} \in\left\{-\frac{1}{2}+\frac{\sqrt{3} i}{2},-\frac{1}{2}-\frac{\sqrt{3} i}{2}, 1,0\right\}$ and $Q_{1}=Q_{2}$,

$\left(b_{2}\right) c_{1}+\left(-\frac{1}{2}+\frac{\sqrt{3} i}{2}\right) c_{2} \in\left\{-\frac{1}{2}+\frac{\sqrt{3} i}{2},-\frac{1}{2}-\frac{\sqrt{3} i}{2}, 1,0\right\}$ and $Q_{1}=\left(-\frac{1}{2}+\frac{\sqrt{3} i}{2}\right) Q_{2}$,

$\left(b_{3}\right) c_{1}+\left(-\frac{1}{2}-\frac{\sqrt{3} i}{2}\right) c_{2} \in\left\{-\frac{1}{2}+\frac{\sqrt{3} i}{2},-\frac{1}{2}-\frac{\sqrt{3} i}{2}, 1,0\right\}$ and $Q_{1}=\left(-\frac{1}{2}-\frac{\sqrt{3} i}{2}\right) Q_{2}$,

$\left(b_{4}\right)\left(c_{j}, c_{k}\right) \in\left\{\begin{array}{l}\left(\frac{1}{2}-\frac{i \sqrt{3}}{2}, \frac{1}{2}+\frac{i \sqrt{3}}{2}\right),\left(\frac{1}{2}-\frac{i \sqrt{3}}{6}, \frac{1}{2}+\frac{i \sqrt{3}}{6}\right),\left(\frac{1}{2}+\frac{i \sqrt{3}}{2},-1\right), \\ \left(\frac{i}{\sqrt{3}}, \frac{-1}{2}+\frac{i \sqrt{3}}{6}\right),\left(-1, \frac{1}{2}-\frac{i \sqrt{3}}{2}\right),\left(\frac{-1}{2}-\frac{i \sqrt{3}}{6}, \frac{-i}{\sqrt{3}}\right),\left(\frac{-i}{\sqrt{3}}, \frac{i}{\sqrt{3}}\right), \\ \left(\frac{1}{2}+\frac{i \sqrt{3}}{6}, \frac{-1}{2}-\frac{i \sqrt{3}}{6}\right),\left(\frac{-1}{2}+\frac{i \sqrt{3}}{6}, \frac{1}{2}-\frac{i \sqrt{3}}{6}\right)\end{array}\right\}$ and $(-1+i \sqrt{3}) Q_{j}+(-2-$

$2 i \sqrt{3}) Q_{j}^{3} Q_{k}+6 Q_{j}^{2} Q_{k}^{2}+(-2+2 i \sqrt{3}) Q_{j} Q_{k}^{3}+(-1-i \sqrt{3}) Q_{k}=\mathbf{0}$,

$\left(b_{5}\right)\left(c_{j}, c_{k}\right) \in\left\{\begin{array}{l}\left(\frac{-1}{2}+\frac{i \sqrt{3}}{2}, \frac{3}{2}-\frac{i \sqrt{3}}{2}\right),\left(\frac{-1}{2}-\frac{i \sqrt{3}}{2}, \frac{3}{2}+\frac{i \sqrt{3}}{2}\right),\left(1, \frac{-3}{2}+\frac{i \sqrt{3}}{2}\right), \\ \left(\frac{-1}{2}-\frac{i \sqrt{3}}{2}, i \sqrt{3}\right),\left(1, \frac{-3}{2}-\frac{i \sqrt{3}}{2}\right),\left(\frac{-1}{2}+\frac{i \sqrt{3}}{2},-i \sqrt{3}\right), \\ (1,-1),\left(\frac{-1}{2}+\frac{i \sqrt{3}}{2}, \frac{1}{2}-\frac{i \sqrt{3}}{2}\right),\left(\frac{-1}{2}-\frac{i \sqrt{3}}{2}, \frac{1}{2}+\frac{i \sqrt{3}}{2}\right)\end{array}\right\}$ and $(6-2 i \sqrt{3}) Q_{j}^{3} Q_{k}-18 Q_{j}^{2} Q_{k}^{2}+$

$$
(18+6 i \sqrt{3}) Q_{j} Q_{k}^{3}+(-6-4 i \sqrt{3}) Q_{k}=\mathbf{0},
$$

$\left(b_{6}\right) c_{1}+c_{2} \in\left\{-\frac{1}{2}+\frac{\sqrt{3} i}{2},-\frac{1}{2}-\frac{\sqrt{3} i}{2}, 1,0\right\}$ and $Q_{1}=Q_{2}$,

$\left(a_{7}\right)\left(c_{j}, c_{k}\right) \in\left\{\begin{array}{l}\left(\frac{1}{2}-\frac{i \sqrt{3}}{2}, \frac{1}{2}-\frac{i \sqrt{3}}{2}\right),\left(\frac{1}{2}+\frac{i \sqrt{3}}{2}, \frac{1}{2}+\frac{i \sqrt{3}}{2}\right),\left(\frac{1}{2}-\frac{i \sqrt{3}}{6}, \frac{-i}{\sqrt{3}}\right), \\ \left(\frac{-1}{2}-\frac{i \sqrt{3}}{6}, \frac{-1}{2}+\frac{i \sqrt{3}}{6}\right),\left(\frac{i}{\sqrt{3}}, \frac{1}{2}+\frac{i \sqrt{3}}{6}\right),(-1,-1), \\ \left(\frac{1}{2}+\frac{i \sqrt{3}}{6}, \frac{i}{\sqrt{3}}\right),\left(\frac{-1}{2}+\frac{i \sqrt{3}}{6}, \frac{-1}{2}-\frac{i \sqrt{3}}{6}\right),\left(\frac{-i}{\sqrt{3}}, \frac{1}{2}-\frac{i \sqrt{3}}{6}\right)\end{array}\right\}$ and $\left(\frac{-5}{9}+\frac{\sqrt{3}}{9}\right) Q_{j}-\frac{4}{9} Q_{j}^{3} Q_{k}+\left(\frac{-1}{3}+\right.$

$\left.\frac{i}{\sqrt{3}}\right) Q_{j}^{2} Q_{k}^{2}+\left(\frac{2}{9}+\frac{2 i \sqrt{3}}{9}\right) Q_{j} Q_{k}^{3}+\left(\frac{1}{9}+\frac{i}{\sqrt{3}}\right) Q_{k}=\mathbf{0}$,

$\left(b_{8}\right)\left(c_{j}, c_{k}\right) \in\left\{\begin{array}{l}\left(\frac{-1}{2}+\frac{i \sqrt{3}}{2}, \frac{-3}{2}-\frac{i \sqrt{3}}{2}\right),\left(\frac{-1}{2}-\frac{i \sqrt{3}}{2},-i \sqrt{3}\right),\left(1, \frac{3}{2}+\frac{i \sqrt{3}}{2}\right), \\ \left(\frac{-1}{2}-\frac{i \sqrt{3}}{2}, \frac{3}{2}-\frac{i \sqrt{3}}{2}\right),(1, i \sqrt{3}),\left(\frac{-1}{2}+\frac{i \sqrt{3}}{2}, \frac{-3}{2}+\frac{i \sqrt{3}}{2}\right), \\ \left(1, \frac{1}{2}+\frac{i \sqrt{3}}{2}\right),\left(\frac{-1}{2}+\frac{i \sqrt{3}}{2},-1\right),\left(\frac{-1}{2}-\frac{i \sqrt{3}}{2}, \frac{1}{2}-\frac{i \sqrt{3}}{2}\right)\end{array}\right\}$ and $(6+2 i \sqrt{3}) Q_{j}^{3} Q_{k}+(9+$

$9 i \sqrt{3}) Q_{j}^{2} Q_{k}^{2}+12 i \sqrt{3} Q_{j} Q_{k}^{3}+(-6+4 i \sqrt{3}) Q_{k}=\mathbf{0}$,

$\left(b_{9}\right) c_{1}+\left(-\frac{1}{2}+\frac{\sqrt{3} i}{2}\right) c_{2} \in\left\{-\frac{1}{2}+\frac{\sqrt{3} i}{2},-\frac{1}{2}-\frac{\sqrt{3} i}{2}, 1,0\right\}$ and $Q_{1}=\left(-\frac{1}{2}+\frac{\sqrt{3} i}{2}\right) Q_{2}$, 


$$
\begin{aligned}
& \left(b_{10}\right)\left(c_{j}, c_{k}\right) \in\left\{\begin{array}{l}
\left(\frac{3}{2}-\frac{i \sqrt{3}}{2}, 1\right),\left(\frac{3}{2}+\frac{i \sqrt{3}}{2}, \frac{-1}{2}+\frac{i \sqrt{3}}{2}\right),\left(i \sqrt{3}, \frac{-1}{2}+\frac{i \sqrt{3}}{2}\right), \\
\left(\frac{-3}{2}+\frac{i \sqrt{3}}{2}, \frac{-1}{2}-\frac{i \sqrt{3}}{2}\right),(-i \sqrt{3}, 1),\left(\frac{-3}{2}-\frac{i \sqrt{3}}{2}, \frac{-1}{2}-\frac{i \sqrt{3}}{2}\right), \\
\left(\frac{1}{2}-\frac{i \sqrt{3}}{2}, 1\right),\left(\frac{1}{2}+\frac{i \sqrt{3}}{2}, \frac{-1}{2}+\frac{i \sqrt{3}}{2}\right),\left(-1, \frac{-1}{2}-\frac{i \sqrt{3}}{2}\right)
\end{array}\right\} \text { and }(9+i \sqrt{3}) Q_{j}+12 i \sqrt{3} Q_{j}^{3} Q_{k}- \\
& 18 Q_{j}^{2} Q_{k}^{2}-4 i \sqrt{3} Q_{j} Q_{k}^{3}=\mathbf{0}, \\
& \left(b_{11}\right) c_{1}+\left(-\frac{1}{2}-\frac{\sqrt{3} i}{2}\right) c_{2} \in\left\{-\frac{1}{2}+\frac{\sqrt{3} i}{2},-\frac{1}{2}-\frac{\sqrt{3} i}{2}, 1,0\right\} \text { and } Q_{1}=\left(-\frac{1}{2}-\frac{\sqrt{3} i}{2}\right) Q_{2}, \\
& \left(b_{12}\right)\left(c_{j}, c_{k}\right) \in\left\{\begin{array}{l}
(1,1),\left(1, \frac{-1}{2}+\frac{i \sqrt{3}}{2}\right),\left(1, \frac{-1}{2}-\frac{i \sqrt{3}}{2}\right),\left(\frac{-1}{2}+\frac{i \sqrt{3}}{2}, 1\right), \\
\left(\frac{-1}{2}+\frac{i \sqrt{3}}{2}, \frac{-1}{2}+\frac{i \sqrt{3}}{2}\right),\left(\frac{-1}{2}+\frac{i \sqrt{3}}{2}, \frac{-1}{2}-\frac{i \sqrt{3}}{2}\right),\left(\frac{-1}{2}-\frac{i \sqrt{3}}{2}, 1\right), \\
\left(\frac{-1}{2}-\frac{i \sqrt{3}}{2}, \frac{-1}{2}+\frac{i \sqrt{3}}{2}\right),\left(\frac{-1}{2}-\frac{i \sqrt{3}}{2}, \frac{-1}{2}-\frac{i \sqrt{3}}{2}\right)
\end{array}\right\} \text { and } 4 Q_{j}^{3} Q_{k}+6 Q_{j}^{2} Q_{k}^{2}+4 Q_{j} Q_{k}^{3}=
\end{aligned}
$$

$\mathbf{0}$,

where $j \neq k$ and $j, k=1,2$.

Since the sets of generalized and hypergeneralized projectors are subsets of the set of quadripotent matrices, the results obtained cover those established in $[3,8,12]$.

\subsection{Tripotency of linear combinations of three tripotent matrices that mutually commute}

Let $T_{i} \in \mathbb{C}_{n}, i=1,2,3$, be nonzero tripotent matrices such that $T_{1} T_{2}=T_{2} T_{1}, T_{1} T_{3}=T_{3} T_{1}$, and $T_{2} T_{3}=$ $T_{3} T_{2}$. It is known that $\sigma\left(T_{i}\right) \subseteq\{1,-1,0\}, i=1,2,3,[15$, Proposition 5.5.21]. The problem considered is to characterize all situations in which a linear combination of the form

$$
c_{1} T_{1}+c_{2} T_{2}+c_{3} T_{3}=T
$$

is a tripotent matrix. In other words, the problem tackled is to characterize all situations in which a linear combination of the form (10) is a matrix such that $\sigma(T) \subseteq\{1,-1,0\}$. By the product rule, there are $3 \cdot 3 \cdot 3=27$ left-hand sides of the linear equations. However, since one of these 27 left-hand sides is $0 c_{1}+0 c_{2}+0 c_{3}$, there are 26 left-hand sides. Besides, since all of the spectra $\sigma(T)$ and $\sigma\left(T_{i}\right), i=1,2,3$, contain all eigenvalues with their opposite signs, it is sufficient to consider $\frac{26}{2}=13$ left-hand sides. Hence, the number of linear systems that need to be solved is $\left(\begin{array}{c}13 \\ 1\end{array}\right)+\left(\begin{array}{c}13 \\ 2\end{array}\right)+\left(\begin{array}{c}13 \\ 3\end{array}\right)=377$. Moreover, direct calculations show that the matrix $T$ of the form (10) is tripotent if and only if

$$
\begin{aligned}
& \left(c_{1}^{3}-c_{1}\right) T_{1}+3 c_{1}^{2} c_{2} T_{1}^{2} T_{2}+3 c_{1} c_{2}^{2} T_{1} T_{2}^{2}+\left(c_{2}^{3}-c_{2}\right) T_{2}+3 c_{1}^{2} c_{3} T_{1}^{2} T_{3}+ \\
& 6 T_{1} T_{2} T_{3}+3 c_{2}^{2} c_{3} T_{2}^{2} T_{3}+3 c_{1} c_{3}^{2} T_{1} T_{3}^{2}+3 c_{2} c_{3}^{2} T_{2} T_{3}^{2}+\left(c_{3}^{3}-c_{3}\right) T_{3}=\mathbf{0} .
\end{aligned}
$$

The equation (11) is utilized in the process of determining the conditions on the matrices in the linear systems whose set of solutions consists of unique solutions.

Before giving the results, let us introduce the $\operatorname{symbol}|\cdot|$ that will be used to express the results, which are minus times of each other, all together in a single item of the theorem. This symbol is used in both the scalars $c_{i}$ and the matrices $T_{i}, i=1,2,3$, and it denotes neither the absolute value of the scalars nor the determinants of the matrices. However, this symbol acts as a real absolute value function. Namely, $\left|c_{i}\right|:= \pm c_{i}$ and $\left|T_{i}\right|:= \pm T_{i}, i=1,2,3$. Nevertheless, the scalars $c_{i}$ and the matrices $T_{i}$, which are indexed with the same number, are positive (or negative) at the same time. To illustrate, let us consider the following expression

$$
\begin{aligned}
& \left(\left|c_{1}\right|,\left|c_{2}\right|,\left|c_{3}\right|\right) \in\{(3,-1,4),(-3,1,-4)\} \text { and } \\
& \left|T_{1}\right|-\left|T_{2}\right|+2\left|T_{3}\right|-3\left|T_{1}\right|\left|T_{2}\right|\left|T_{3}\right|=\mathbf{0} .
\end{aligned}
$$

Expression (12) consists of the combination of the results listed in the following Table. 


\begin{tabular}{llrr}
\hline 1) $\left(c_{1}, c_{2}, c_{3}\right) \in\{(3,-1,4),(-3,1,-4)\}$ & $2) \quad\left(-c_{1},-c_{2},-c_{3}\right)$ & $\in$ \\
and $T_{1}-T_{2}+2 T_{3}-3 T_{1} T_{2} T_{3}=\mathbf{0}$ & $\{(3,-1,4),(-3,1,-4)\}$ & and \\
& $-T_{1}+T_{2}-2 T_{3}+3 T_{1} T_{2} T_{3}=\mathbf{0}$ & \\
3) $\left(-c_{1}, c_{2}, c_{3}\right) \in\{(3,-1,4),(-3,1,-4)\}$ & $4) \quad\left(c_{1},-c_{2},-c_{3}\right)$ & $\epsilon$ \\
and $-T_{1}-T_{2}+2 T_{3}+3 T_{1} T_{2} T_{3}=\mathbf{0}$ & $\{(3,-1,4),(-3,1,-4)\}$ & and \\
& $T_{1}+T_{2}-2 T_{3}-3 T_{1} T_{2} T_{3}=\mathbf{0}$ & \\
5) $\left(c_{1},-c_{2}, c_{3}\right) \in\{(3,-1,4),(-3,1,-4)\}$ & $6) \quad\left(-c_{1}, c_{2},-c_{3}\right)$ & $\in$ \\
and $T_{1}+T_{2}+2 T_{3}+3 T_{1} T_{2} T_{3}=\mathbf{0}$ & $\{(3,-1,4),(-3,1,-4)\}$ & and \\
& $-T_{1}-T_{2}-2 T_{3}-3 T_{1} T_{2} T_{3}=\mathbf{0}$ & \\
7) $\left(c_{1}, c_{2},-c_{3}\right) \in\{(3,-1,4),(-3,1,-4)\}$ & $8) \quad\left(-c_{1},-c_{2}, c_{3}\right)$ & $\in$ \\
and $T_{1}-T_{2}-2 T_{3}+3 T_{1} T_{2} T_{3}=\mathbf{0}$ & $\{(3,-1,4),(-3,1,-4)\}$ & and \\
& $-T_{1}+T_{2}+2 T_{3}-3 T_{1} T_{2} T_{3}=\mathbf{0}$ & \\
\hline
\end{tabular}

Note that the results 1), 3), 5), and 7) are the same as the results 2), 4), 6), and 8), respectively.

Theorem 6. Let $T_{i} \in \mathbb{C}_{n}, i=1,2,3$, be nonzero tripotent matrices that mutually commute. Let $T$ be a linear combination of the form (10) with $c_{i} \in \mathbb{C} \backslash\{0\}, i=1,2,3$. Then $T$ is tripotent if and only if:

$\left(d_{1}\right)\left|c_{1}\right|+\left|c_{2}\right|+\left|c_{3}\right| \in\{1,-1,0\}$ and $\left|T_{1}\right|=\left|T_{2}\right|=\left|T_{3}\right|$,

$\left(d_{2}\right)\left(c_{i}+\left|c_{j}\right|, c_{k}\right) \in\left\{ \pm(0,1), \pm\left(\frac{1}{2}, \frac{1}{2}\right), \pm\left(\frac{1}{2},-\frac{1}{2}\right)\right\}$ and $T_{i}=\left|T_{j}\right| \neq T_{k}$,

$\left(d_{3}\right)\left(c_{i}+\left|c_{j}\right|,\left|c_{k}\right|\right) \in\{ \pm(-1,2), \pm(0,1), \pm(1,-1)\}$ and $T_{i}=\left|T_{j}\right| \neq\left|T_{k}\right|$,

$\left(d_{4}\right)\left(\left|c_{1}\right|+\frac{1}{2} c_{3},\left|c_{2}\right|+\frac{1}{2} c_{3}\right) \in\left\{ \pm(1,0), \pm(0,1), \pm\left(\frac{1}{2}, \frac{1}{2}\right), \pm\left(\frac{1}{2},-\frac{1}{2}\right),(0,0)\right\}$ and $\frac{1}{2}\left|T_{1}\right|+\frac{1}{2}\left|T_{2}\right|=T_{3}$,

$\left(d_{5}\right)\left(\left|c_{p}\right|+c_{3},\left|c_{q}\right|+2 c_{3}\right) \in\{ \pm(1,0), \pm(-1,2), \pm(0,1), \pm(1,-1),(0,0)\}$ and $\left|T_{p}\right|+2\left|T_{q}\right|=T_{3}$,

$\left(d_{6}\right)\left(c_{i}+\left|c_{j}\right|, c_{k}\right) \in\{ \pm(0,1), \pm(2,-1) \pm(-1,1)\}$ and $T_{i}=\left|T_{j}\right| \neq T_{k}$,

$\left(d_{7}\right)\left(\left|c_{p}\right|+c_{3},\left|c_{q}\right|+c_{3}\right) \in\{ \pm(1,0), \pm(-1,2), \pm(0,1), \pm(1,-1),(0,0)\}$ and $\left|T_{p}\right|+\left|T_{q}\right|=T_{3}$,

$\left(d_{8}\right)\left(c_{i}+\left|c_{j}\right|, c_{k}\right) \in\{ \pm(1,1), \pm(1,-1), \pm(0,1)\}$ and $T_{i}=\left|T_{j}\right| \neq T_{k}$,

$\left(d_{9}\right)\left(\left|c_{1}\right|+c_{3},\left|c_{2}\right|+c_{3}\right) \in\{ \pm(1,1), \pm(1,-1), \pm(1,0), \pm(0,1),(0,0)\}$ and $\left|T_{1}\right|+\left|T_{2}\right|=T_{3}$,

$\left(d_{10}\right)\left(c_{i}+\left|c_{j}\right|, c_{k}\right) \in\{ \pm(0,1)\}$ and $T_{i}=\left|T_{j}\right| \neq T_{k}$,

$\left(d_{11}\right)\left(\left|c_{1}\right|,\left|c_{2}\right|,\left|c_{3}\right|\right) \in\left\{ \pm(-1,1,1), \pm\left(-\frac{1}{2}, \frac{1}{2}, 1\right), \pm\left(-\frac{1}{2}, 1, \frac{1}{2}\right), \pm\left(1,-\frac{1}{2},-\frac{1}{2},\right)\right\}$ and $T_{1}^{2}\left|T_{2}\right|-\left|T_{1}\right| T_{2}^{2}+$ $T_{1}^{2}\left|T_{3}\right|-2\left|T_{1}\right|\left|T_{2}\right|\left|T_{3}\right|+T_{2}^{2}\left|T_{3}\right|-\left|T_{1}\right| T_{3}^{2}+\left|T_{2}\right| T_{3}^{2}=\mathbf{0}$,

$\left(d_{12}\right)\left(\left|c_{i}\right|,\left|c_{j}\right|,\left|c_{k}\right|\right) \in\left\{\begin{array}{l} \pm\left(\frac{1}{2},-\frac{1}{2}, 1\right), \pm\left(-\frac{1}{2}, \frac{1}{2}, 1\right), \pm\left(\frac{3}{4},-\frac{1}{4}, \frac{1}{2}\right), \pm\left(-\frac{1}{4}, \frac{3}{4}, \frac{1}{2}\right), \\ \pm\left(\frac{1}{4}, \frac{1}{4}, \frac{1}{2}\right), \pm\left(\frac{3}{4},-\frac{1}{4},-\frac{1}{2}\right), \pm\left(-\frac{1}{4}, \frac{3}{4},-\frac{1}{2}\right), \pm\left(\frac{1}{4}, \frac{1}{4},-\frac{1}{2}\right)\end{array}\right\}$ and $-\frac{3}{8}\left|T_{i}\right|-\frac{3}{8} T_{i}^{2}\left|T_{j}\right|+$ $\frac{3}{8}\left|T_{i}\right| T_{j}^{2}+\frac{3}{8}\left|T_{j}\right|+\frac{3}{4} T_{i}^{2}\left|T_{k}\right|-\frac{3}{2}\left|T_{i}\right|\left|T_{j}\right|\left|T_{k}\right|+\frac{3}{4} T_{j}^{2}\left|T_{k}\right|+\frac{3}{2}\left|T_{i}\right| T_{k}^{2}-\frac{3}{2}\left|T_{j}\right| T_{k}^{2}=\mathbf{0}$,

$\left(d_{13}\right)\left(\left|c_{i}\right|,\left|c_{j}\right|,\left|c_{k}\right|\right) \in\left\{\begin{array}{l} \pm(-2,2,1), \pm(-1,1,1), \pm\left(-\frac{3}{2}, 2, \frac{1}{2}\right), \\ \pm\left(-\frac{1}{2}, 1, \frac{1}{2}\right), \pm\left(\frac{3}{2},-1,-\frac{1}{2}\right), \pm\left(\frac{1}{2},-1, \frac{1}{2}\right)\end{array}\right\}$ and $-\left|T_{i}\right|+4 T_{i}^{2}\left|T_{j}\right|-4\left|T_{i}\right| T_{j}^{2}+\left|T_{j}\right|+$ $2 T_{i}^{2}\left|T_{k}\right|-4\left|T_{i}\right|\left|T_{j}\right|\left|T_{k}\right|+2 T_{j}^{2}\left|T_{k}\right|-\left|T_{i}\right| T_{k}^{2}+\left|T_{j}\right| T_{k}^{2}=\mathbf{0}$,

$\left(d_{14}\right)\left(\left|c_{i}\right|,\left|c_{j}\right|,\left|c_{k}\right|\right) \in\left\{\begin{array}{l} \pm(1,-1,1), \pm(-1,1,1), \pm\left(1,-\frac{1}{2}, \frac{1}{2}\right), \\ \pm\left(-1, \frac{3}{2}, \frac{1}{2}\right), \pm\left(1,-\frac{3}{2}, \frac{1}{2}\right), \pm\left(-1, \frac{1}{2}, \frac{1}{2}\right)\end{array}\right\}$ and $-T_{i}^{2}\left|T_{j}\right|+\left|T_{i}\right| T_{j}^{2}+T_{i}^{2}\left|T_{k}\right|-$ $2\left|T_{i}\right|\left|T_{j}\right|\left|T_{k}\right|+T_{j}^{2}\left|T_{k}\right|+\left|T_{i}\right| T_{k}^{2}-\left|T_{j}\right| T_{k}^{2}=\mathbf{0}$,

$\left(d_{15}\right)\left(\left|c_{i}\right|,\left|c_{j}\right|,\left|c_{k}\right|\right) \in\left\{ \pm\left(\frac{1}{2},-\frac{1}{2}, 1\right), \pm\left(-\frac{1}{2}, \frac{1}{2}, 1\right), \pm\left(\frac{1}{2}, \frac{1}{2},-2\right), \pm\left(\frac{1}{2}, \frac{1}{2},-1\right)\right\}$ and $\frac{3}{8}\left|T_{i}\right|-\frac{3}{8} T_{i}^{2}\left|T_{j}\right|-$ $\frac{3}{8}\left|T_{i}\right| T_{j}^{2}+\frac{3}{8}\left|T_{j}\right|+\frac{3}{2} T_{i}^{2}\left|T_{k}\right|+3\left|T_{i}\right|\left|T_{j}\right|\left|T_{k}\right|+\frac{3}{2} T_{j}^{2}\left|T_{k}\right|-6\left|T_{i}\right| T_{k}^{2}-6\left|T_{j}\right| T_{k}^{2}+6\left|T_{k}\right|=\mathbf{0}$,

$\left(d_{16}\right)\left(\left|c_{i}\right|,\left|c_{j}\right|,\left|c_{k}\right|\right) \in\left\{\begin{array}{l} \pm(-3,2,2), \pm(-2,1,2), \pm(-2,2,1), \\ \pm(-1,1,1), \pm(2,-1,-1)\end{array}\right\}$ and $-4\left|T_{i}\right|+9 T_{i}^{2}\left|T_{j}\right|-6|X|_{i} T_{j}^{2}+\left|T_{j}\right|+$ $9 T_{i}^{2}\left|T_{k}\right|-12\left|T_{i}\right|\left|T_{j}\right|\left|T_{k}\right|+4 T_{j}^{2}\left|T_{k}\right|-6\left|T_{i}\right| T_{k}^{2}+4\left|T_{j}\right| T_{k}^{2}+\left|T_{k}\right|=\mathbf{0}$,

$\left(d_{17}\right)\left(\left|c_{i}\right|,\left|c_{j}\right|,\left|c_{k}\right|\right) \in\left\{\begin{array}{l} \pm(3,-4,2), \pm(1,-2,2), \pm(2,-3,2), \pm(2,-2,1), \\ \pm(1,-1,1), \pm(2,-3,1), \pm(1,-2,1)\end{array}\right\}$ and $4\left|T_{i}\right|-18 T_{i}^{2}\left|T_{j}\right|+$ $24\left|T_{i}\right| T_{j}^{2}-10\left|T_{j}\right|+9 T_{i}^{2}\left|T_{k}\right|-24\left|T_{i}\right|\left|T_{j}\right|\left|T_{k}\right|+16 T_{j}^{2}\left|T_{k}\right|+6\left|T_{i}\right| T_{k}^{2}-8\left|T_{j}\right| T_{k}^{2}+\left|T_{k}\right|=\mathbf{0}$,

$\left(d_{18}\right)\left(\left|c_{i}\right|,\left|c_{j}\right|,\left|c_{k}\right|\right) \in\{ \pm(1,-2,2), \pm(1,-1,1), \pm(-1,1,1), \pm(1,-2,1)\}$ and $-T_{i}^{2}\left|T_{j}\right|+2\left|T_{i}\right| T_{j}^{2}-\left|T_{j}\right|+$ $T_{i}^{2}\left|T_{k}\right|-4\left|T_{i}\right|\left|T_{j}\right|\left|T_{k}\right|+4 T_{j}^{2}\left|T_{k}\right|+2\left|T_{i}\right| T_{k}^{2}-4\left|T_{j}\right| T_{k}^{2}+\left|T_{k}\right|=\mathbf{0}$,

$\left(d_{19}\right)\left(c_{i}+\left|c_{j}\right|, c_{k}\right) \in\{ \pm(0,1), \pm(1,-1)\}$ and $T_{i}=\left|T_{j}\right| \neq T_{k}$, 


$$
\begin{aligned}
& \left(d_{20}\right)\left(\left|c_{i}\right|,\left|c_{j}\right|,\left|c_{k}\right|\right) \in\left\{\begin{array}{l} 
\pm\left(\frac{1}{3},-\frac{2}{3}, \frac{4}{3}\right), \pm\left(\frac{2}{3},-\frac{1}{3}, \frac{2}{3}\right), \pm\left(\frac{1}{3}, \frac{4}{3},-\frac{2}{3}\right), \pm\left(-\frac{1}{3}, \frac{2}{3}, \frac{2}{3}\right), \\
\pm\left(\frac{2}{3}, \frac{2}{3},-\frac{1}{3}\right), \pm\left(\frac{1}{3}, \frac{1}{3}, \frac{1}{3}\right), \pm\left(\frac{1}{3},-\frac{2}{3}, \frac{1}{3}\right), \pm\left(-\frac{2}{3}, \frac{1}{3}, \frac{1}{3}\right), \\
\pm\left(\frac{1}{3}, \frac{1}{3},-\frac{2}{3}\right)
\end{array}\right\} \text { and }-\frac{8}{27}\left|T_{i}\right|-\frac{2}{9} T_{i}^{2}\left|T_{j}\right|+ \\
& \frac{4}{9}\left|T_{i}\right| T_{j}^{2}+\frac{10}{27}\left|T_{j}\right|+\frac{4}{9} T_{i}^{2}\left|T_{k}\right|-\frac{16}{9}\left|T_{i}\right|\left|T_{j}\right|\left|T_{k}\right|+\frac{16}{9} T_{j}^{2}\left|T_{k}\right|+\frac{16}{9}\left|T_{i}\right| T_{k}^{2}-\frac{32}{9}\left|T_{j}\right| T_{k}^{2}+\frac{28}{27}\left|T_{k}\right|=\mathbf{0}, \\
& \left(d_{21}\right)\left(\left|c_{i}\right|,\left|c_{j}\right|,\left|c_{k}\right|\right) \in\left\{\begin{array}{c} 
\pm(1,2,-2), \pm(1,1,-1), \pm(1,2,-4), \\
\pm(1,1,-3), \pm(1,2,-3), \pm(1,1,-2)
\end{array}\right\} \text { and }-T_{i}^{2}\left|T_{j}\right|-2\left|T_{i}\right| T_{j}^{2}-\left|T_{j}\right|+2 T_{i}^{2}\left|T_{k}\right|+ \\
& 8\left|T_{i}\right|\left|T_{j}\right|\left|T_{k}\right|+8 T_{j}^{2}\left|T_{k}\right|-8\left|T_{i}\right| T_{k}^{2}-16\left|T_{j}\right| T_{k}^{2}+10\left|T_{k}\right|=\mathbf{0}, \\
& \left(d_{22}\right)\left(\left|c_{i}\right|,\left|c_{j}\right|,\left|c_{k}\right|\right) \in\left\{\begin{array}{l} 
\pm\left(\frac{1}{2},-\frac{1}{2}, 1\right), \pm\left(\frac{3}{2}, \frac{1}{2},-1\right), \pm\left(-\frac{1}{2}, \frac{1}{2}, 1\right), \\
\pm\left(\frac{1}{2}, \frac{3}{2},-1\right), \pm(1,1,-1), \pm\left(\frac{1}{2}, \frac{1}{2},-1\right)
\end{array}\right\} \text { and }-\frac{3}{8}\left|T_{i}\right|-\frac{3}{8} T_{i}^{2}\left|T_{j}\right|+\frac{3}{8}\left|T_{i}\right| T_{j}^{2}+ \\
& \frac{3}{8}\left|T_{j}\right|+\frac{3}{4} T_{i}^{2}\left|T_{k}\right|-\frac{3}{2}\left|T_{i}\right|\left|T_{j}\right|\left|T_{k}\right|+\frac{3}{4} T_{j}^{2}\left|T_{k}\right|+\frac{3}{2}\left|T_{i}\right| T_{k}^{2}-\frac{3}{2}\left|T_{j}\right| T_{k}^{2}=\mathbf{0}, \\
& \left(d_{23}\right)\left(\left|c_{i}\right|,\left|c_{j}\right|,\left|c_{k}\right|\right) \in\left\{\begin{array}{l} 
\pm(1,1,-1), \pm(1,-1,1), \pm(-1,1,1), \\
\pm(1,1,-3) \pm(1,1,-2)
\end{array}\right\} \text { and } T_{i}^{2}\left|T_{j}\right|+\left|T_{i}\right| T_{j}^{2}-T_{i}^{2}\left|T_{k}\right|- \\
& 2\left|T_{i}\right|\left|T_{j}\right|\left|T_{k}\right|-T_{j}^{2}\left|T_{k}\right|+\left|T_{i}\right| T_{k}^{2}+\left|T_{j}\right| T_{k}^{2}=\mathbf{0}, \\
& \left(d_{24}\right)\left(\left|c_{i}\right|,\left|c_{j}\right|,\left|c_{k}\right|\right) \in\left\{\begin{array}{l} 
\pm\left(\frac{1}{2}, \frac{1}{2}, \frac{1}{2}\right), \pm\left(\frac{1}{2}, \frac{1}{2},-\frac{3}{2}\right), \pm\left(\frac{1}{2}, \frac{1}{2},-\frac{1}{2}\right), \\
\pm\left(\frac{1}{2},-\frac{1}{2}, \frac{1}{2}\right), \pm\left(-\frac{1}{2}, \frac{1}{2}, \frac{3}{2}\right), \pm\left(-\frac{1}{2}, \frac{1}{2}, \frac{1}{2}\right)
\end{array}\right\} \text { and }-\frac{3}{8}\left|T_{i}\right|+\frac{3}{8} T_{i}^{2}\left|T_{j}\right|+\frac{3}{8}\left|T_{i}\right| T_{j}^{2}-\frac{3}{8}\left|T_{j}\right|+ \\
& \frac{3}{8} T_{i}^{2}\left|T_{k}\right|+\frac{3}{4}\left|T_{i}\right|\left|T_{j}\right|\left|T_{k}\right|+\frac{3}{8} T_{j}^{2}\left|T_{k}\right|+\frac{3}{8}\left|T_{i}\right| T_{k}^{2}+\frac{3}{8}\left|T_{j}\right| T_{k}^{2}-\frac{3}{8}\left|T_{k}\right|=\mathbf{0}, \\
& \left(d_{25}\right)\left(c_{i}, c_{j}, c_{k}\right) \in\left\{ \pm\left(\frac{1}{2}, \frac{1}{2}, 1\right), \pm\left(\frac{1}{2}, \frac{1}{2},-1\right), \pm\left(\frac{1}{2},-\frac{1}{2}, 1\right), \pm\left(-\frac{1}{2}, \frac{1}{2}, 1\right)\right\} \text { and }-\frac{3}{8} T_{i}+\frac{3}{8} T_{i}^{2} T_{j}+\frac{3}{8} T_{i} T_{j}^{2}-\frac{3}{8} T_{j}+ \\
& \frac{3}{4} T_{i}^{2} T_{k}+\frac{3}{2} T_{i} T_{j} T_{k}+\frac{3}{4} T_{j}^{2} T_{k}+\frac{3}{2} T_{i} T_{k}^{2}+\frac{3}{2} T_{j} T_{k}^{2}=\mathbf{0} \\
& \left(d_{26}\right)\left(\left|c_{i}\right|,\left|c_{j}\right|,\left|c_{k}\right|\right) \in\{ \pm(-1,2,2), \pm(-1,2,1), \pm(-1,1,2), \pm(-1,1,1)\} \text { and } T_{i}^{2}\left|T_{j}\right|-2\left|T_{i}\right| T_{j}^{2}+\left|T_{j}\right|+ \\
& T_{i}^{2}\left|T_{k}\right|-4\left|T_{i}\right|\left|T_{j}\right|\left|T_{k}\right|+4 T_{j}^{2}\left|T_{k}\right|-2\left|T_{i}\right| T_{k}^{2}+4\left|T_{j}\right| T_{k}^{2}+\left|T_{k}\right|=\mathbf{0}, \\
& \left(d_{27}\right)\left(\left|c_{1}\right|,\left|c_{2}\right|,\left|c_{3}\right|\right) \in\left\{\begin{array}{l} 
\pm\left(\frac{1}{2}, \frac{1}{2}, \frac{1}{2}\right), \pm\left(\frac{1}{2}, \frac{1}{2},-\frac{3}{2}\right), \pm\left(\frac{1}{2}, \frac{1}{2},-\frac{1}{2}\right), \pm\left(\frac{1}{2},-\frac{3}{2}, \frac{1}{2}\right), \\
\pm\left(-\frac{3}{2}, \frac{1}{2}, \frac{1}{2}\right), \pm\left(\frac{1}{2},-\frac{1}{2}, \frac{1}{2}\right), \pm\left(-\frac{1}{2}, \frac{1}{2}, \frac{1}{2}\right)
\end{array}\right\} \text { and } \frac{15}{8}\left|T_{1}\right|-\frac{27}{8} T_{1}^{2}\left|T_{2}\right|+ \\
& \frac{9}{8}\left|T_{1}\right| T_{2}^{2}+\frac{3}{8}\left|T_{2}\right|-\frac{27}{8} T_{1}^{2}\left|T_{3}\right|+\frac{9}{4}\left|T_{1}\right|\left|T_{2}\right|\left|T_{3}\right|-\frac{3}{8} T_{2}^{2}\left|T_{3}\right|+\frac{9}{8}\left|T_{1}\right| T_{3}^{2}-\frac{3}{8}\left|T_{2}\right| T_{3}^{2}+\frac{3}{8}\left|T_{3}\right|=\mathbf{0}, \\
& \left(d_{28}\right)\left(\left|c_{1}\right|+c_{3},\left|c_{2}\right|+c_{3}\right) \in\{ \pm(1,0), \pm(0,1), \pm(1,-1),(0,0)\} \text { and }\left|T_{1}\right|+\left|T_{2}\right|=T_{3} \text {, } \\
& \left(d_{29}\right)\left(\left|c_{i}\right|,\left|c_{j}\right|,\left|c_{k}\right|\right) \in\left\{\begin{array}{l} 
\pm(-2,1,3), \pm(-2,1,1), \pm(-2,1,2), \\
\pm(-1,1,2), \pm(-1,1,1)
\end{array}\right\} \text { and } 2\left|T_{i}\right|-4 T_{i}^{2}\left|T_{j}\right|+2\left|T_{i}\right| T_{j}^{2}-4 T_{i}^{2}\left|T_{k}\right|+ \\
& 4\left|T_{i}\right|\left|T_{j}\right|\left|T_{k}\right|-T_{j}^{2}\left|T_{k}\right|+2\left|T_{i}\right| T_{k}^{2}-\left|T_{j}\right| T_{k}^{2}=\mathbf{0}, \\
& \left(d_{30}\right)\left(\left|c_{i}\right|,\left|c_{j}\right|,\left|c_{k}\right|\right) \in\{ \pm(-1,2,1), \pm(1,-2,1), \pm(1,-1,1), \pm(-1,1,1)\} \text { and } 2 T_{i}^{2}\left|T_{j}\right|-4\left|T_{i}\right| T_{j}^{2}+2\left|T_{j}\right|+ \\
& T_{i}^{2}\left|T_{k}\right|-4\left|T_{i}\right|\left|T_{j}\right|\left|T_{k}\right|+4 T_{j}^{2}\left|T_{k}\right|-\left|T_{i}\right| T_{k}^{2}+\left|T_{j}\right| T_{k}^{2}=\mathbf{0}, \\
& \left(d_{31}\right)\left(c_{1}, c_{2}, c_{3}\right) \in\{ \pm(1,1,1), \pm(1,1,-1), \pm(1,-1,1), \pm(-1,1,1)\} \text { and } T_{1}^{2} T_{2}+T_{1} T_{2}^{2}+T_{1}^{2} T_{3}+2 T_{1} T_{2} T_{3}+ \\
& T_{2}^{2} T_{3}+T_{1} T_{3}^{2}+T_{2} T_{3}^{2}=\mathbf{0} \text {, }
\end{aligned}
$$

where $i \neq j, i \neq k, j \neq k, p \neq q$ and $i, j, k=1,2,3$ and $p, q=1,2$.

The results obtained are an expanding of the results given in [5] and cover those established in [22, 30].

\subsection{Tripotency of linear combinations of four involutive matrices that mutually commute}

Let $P_{i} \in \mathbb{C}_{n}, i=1,2,3,4$, be nonzero involutive matrices that mutually commute. It is known that $\sigma\left(P_{i}\right) \subseteq$ $\{1,-1\}, i=1,2,3,4$, [15, Proposition 5.5.21]. The problem considered is to characterize all situations in which a linear combination of the form

$$
c_{1} P_{1}+c_{2} P_{2}+c_{3} P_{3}+c_{4} P_{4}=P
$$

is a tripotent matrix. By the product rule, there are $2 \cdot 2 \cdot 2 \cdot 2=16$ left-hand sides of the linear equations. However, since all of the spectra $\sigma(P)$ and $\sigma\left(P_{i}\right), i=1,2,3,4$, contain all eigenvalues with their opposite signs, it is sufficient to consider $\frac{16}{2}=8$ left-hand sides. Hence, the number of linear systems that need to be solved is $\left(\begin{array}{l}8 \\ 1\end{array}\right)+\left(\begin{array}{l}8 \\ 2\end{array}\right)+\left(\begin{array}{l}8 \\ 3\end{array}\right)+\left(\begin{array}{l}8 \\ 4\end{array}\right)=162$. Moreover, direct calculations show that the matrix $P$ of the form (13) is 
tripotent if and only if

$$
\begin{aligned}
& \left(c_{1}^{3}+3 c_{1} c_{2}^{2}+3 c_{1} c_{3}^{2}+3 c_{1} c_{4}^{2}-c_{1}\right) P_{1}+\left(c_{2}^{3}+3 c_{1}^{2} c_{2}+3 c_{2} c_{3}^{2}+3 c_{2} c_{4}^{2}-c_{2}\right) P_{2}+ \\
& \left(c_{3}^{3}+3 c_{1}^{2} c_{3}+3 c_{2}^{2} c_{3}+3 c_{3} c_{4}^{2}-c_{3}\right) P_{3}+\left(c_{4}^{3}+3 c_{1}^{2} c_{4}+3 c_{2}^{2} c_{4}+3 c_{3}^{2} c_{4}-c_{4}\right) P_{4}+ \\
& 6 c_{1} c_{2} c_{3} P_{1} P_{2} P_{3}+6 c_{1} c_{2} c_{4} P_{1} P_{2} P_{4}+6 c_{1} c_{3} c_{4} P_{1} P_{3} P_{4}+6 c_{2} c_{3} c_{4} P_{2} P_{3} P_{4}=\mathbf{0} .
\end{aligned}
$$

The equation (14) is utilized in the process of determining the conditions on the matrices in the linear systems whose set of solutions consists of unique solutions.

Theorem 7. Let $P_{i} \in \mathbb{C}_{n}, i=1,2,3$, 4, be nonzero involutive matrices that mutually commute. Let $P$ be a linear combination of the form (13) with $c_{i} \in \mathbb{C} \backslash\{0\}$. Then $P$ is tripotent if and only if:

$$
\left(e_{1}\right)\left|c_{1}\right|+\left|c_{2}\right|+\left|c_{3}\right|+\left|c_{4}\right| \in\{1,-1,0\} \text { and }\left|P_{1}\right|=\left|P_{2}\right|=\left|P_{3}\right|=\left|P_{4}\right| \text {, }
$$$$
\left(e_{2}\right)\left(c_{i}, c_{j}+\left|c_{k}\right|+\left|c_{l}\right|\right) \in\left\{ \pm(1,0), \pm\left(\frac{1}{2}, \frac{1}{2}\right), \pm\left(\frac{1}{2},-\frac{1}{2}\right)\right\} \text { and } P_{i} \neq P_{j}=\left|P_{k}\right|=\left|P_{l}\right| \text {, where } j<k \text { and } j<l \text {, }
$$$$
\left(e_{3}\right)\left(c_{i}+\left|c_{j}\right|, c_{k}+\left|c_{l}\right|\right) \in\left\{ \pm(1,0), \pm(0,1), \pm\left(\frac{1}{2}, \frac{1}{2}\right), \pm\left(\frac{1}{2},-\frac{1}{2}\right),(0,0)\right\} \text { and } P_{i}=\left|P_{j}\right| \neq P_{k}=\left|P_{l}\right| \text {, where } i<j
$$
and $k<l$,

$\left(e_{4}\right)\left(\left|c_{i}\right|+\left|c_{j}\right|,\left|c_{k}\right|,\left|c_{l}\right|\right) \in\left\{\begin{array}{l} \pm(-1,1,1), \pm\left(-\frac{1}{2}, \frac{1}{2}, 1\right), \pm\left(-\frac{1}{2}, 1, \frac{1}{2}\right), \\ \pm\left(0, \frac{1}{2}, \frac{1}{2}\right), \pm\left(0, \frac{1}{2},-\frac{1}{2}\right), \pm\left(-1, \frac{1}{2}, \frac{1}{2}\right)\end{array}\right\}$ and $\left|P_{i}\right|=\left|P_{j}\right| \neq\left|P_{k}\right| \neq\left|P_{l}\right|$, where $i<j$,

$\left(e_{5}\right)\left(\left|c_{1}\right|+c_{4},\left|c_{2}\right|+c_{4},\left|c_{3}\right|+c_{4}\right) \in\left\{\begin{array}{l} \pm(1,0,0), \pm(0,1,0), \pm\left(\frac{1}{2}, \frac{1}{2}, 0\right), \pm(0,0,1), \\ \pm(-1,1,1), \pm\left(-\frac{1}{2}, \frac{1}{2}, 1\right), \pm\left(\frac{1}{2}, 0, \frac{1}{2}\right), \pm\left(-\frac{1}{2}, 1, \frac{1}{2}\right), \\ \pm\left(0, \frac{1}{2}, \frac{1}{2}\right), \pm\left(-\frac{1}{2}, 0, \frac{1}{2}\right), \pm\left(0, \frac{1}{2},-\frac{1}{2}\right), \pm\left(-1, \frac{1}{2}, \frac{1}{2}\right), \\ \pm\left(\frac{1}{2},-\frac{1}{2}, 0\right),(0,0,0)\end{array}\right\}$ and $\left|P_{1}\right|+$ $\left|P_{2}\right|+\left|P_{3}\right|=P_{4}$,

$\left(e_{6}\right)\left(c_{i}+\left|c_{j}\right|, c_{k}, c_{l}\right) \in\left\{ \pm\left(0, \frac{1}{2}, \frac{1}{2}\right), \pm\left(0, \frac{1}{2},-\frac{1}{2}\right)\right\}$ and $P_{i}=\left|P_{j}\right| \neq\left|P_{k}\right| \neq\left|P_{l}\right|$, where $i<j$,

$\left(e_{7}\right)\left(\left|c_{i}\right|,\left|c_{j}\right|,\left|c_{k}\right|,\left|c_{l}\right|\right) \in\left\{\begin{array}{l} \pm(-2,1,1,1), \pm\left(-\frac{3}{2}, \frac{1}{2}, 1,1\right), \pm\left(-\frac{3}{2}, 1, \frac{1}{2}, 1\right), \\ \pm\left(-1, \frac{1}{2}, \frac{1}{2}, 1\right), \pm\left(-\frac{3}{2}, 1,1, \frac{1}{2}\right), \pm\left(-1, \frac{1}{2}, 1, \frac{1}{2}\right), \\ \pm\left(-1,1, \frac{1}{2}, \frac{1}{2}\right), \pm\left(-\frac{1}{2}, \frac{1}{2}, \frac{1}{2}, \frac{1}{2}\right), \pm\left(\frac{1}{2}, \frac{1}{2},-\frac{1}{2},-\frac{1}{2}\right), \\ \pm\left(\frac{1}{2},-\frac{1}{2}, \frac{1}{2},-\frac{1}{2}\right), \pm\left(-\frac{1}{2}, \frac{1}{2}, \frac{1}{2},-\frac{1}{2}\right), \pm\left(-\frac{3}{2}, \frac{1}{2}, \frac{1}{2}, \frac{1}{2}\right)\end{array}\right\}$ and $-4\left|P_{i}\right|+3\left|P_{j}\right|+$ $3\left|P_{k}\right|+3\left|P_{l}\right|-2\left|P_{i}\right|\left|P_{j}\right|\left|P_{k}\right|-2\left|P_{i}\right|\left|P_{j}\right|\left|P_{l}\right|-2\left|P_{i}\right|\left|P_{k}\right|\left|P_{l}\right|+\left|P_{j}\right|\left|P_{k}\right|\left|P_{l}\right|=\mathbf{0}$,

$\left(e_{8}\right)\left(\left|c_{i}\right|,\left|c_{j}\right|,\left|c_{k}\right|,\left|c_{l}\right|\right) \in\left\{\begin{array}{l} \pm\left(-\frac{1}{2},-\frac{1}{2}, 1,1\right), \pm\left(\frac{1}{2},-1, \frac{1}{2}, 1\right), \pm\left(-1, \frac{1}{2}, 1, \frac{1}{2}\right), \\ \pm\left(\frac{1}{2},-\frac{1}{2}, \frac{1}{2}, \frac{1}{2}\right), \pm\left(-\frac{1}{2}, \frac{1}{2}, \frac{1}{2}, \frac{1}{2}\right), \pm\left(\frac{1}{2}, \frac{1}{2},-\frac{1}{2},-\frac{1}{2}\right), \\ \pm\left(-1,1, \frac{1}{2},-\frac{1}{2}\right), \pm\left(-\frac{1}{2}, \frac{1}{2}, \frac{1}{2},-\frac{1}{2}\right)\end{array}\right\}$ and $-3 P_{1}-3 P_{2}+\frac{9}{2} P_{3}+$ $\frac{9}{2} P_{4}+\frac{3}{2} P_{1} P_{2} P_{3}+\frac{3}{2} P_{1} P_{2} P_{4}-3 P_{1} P_{3} P_{4}-3 P_{2} P_{3} P_{4}=\mathbf{0}$,

$\left(e_{9}\right)\left(c_{1}, c_{2}, c_{3},\left|c_{4}\right|\right) \in\left\{\begin{array}{l} \pm\left(\frac{1}{2}, \frac{1}{2}, \frac{1}{2},-\frac{1}{2}\right), \pm\left(\frac{3}{4}, \frac{1}{4}, \frac{1}{4},-\frac{1}{4}\right), \pm\left(\frac{1}{2}, \frac{1}{2},-\frac{1}{2}, \frac{1}{2}\right), \pm\left(\frac{1}{4}, \frac{3}{4},-\frac{1}{4}, \frac{1}{4}\right), \\ \pm\left(\frac{3}{4}, \frac{1}{4},-\frac{1}{4}, \frac{1}{4}\right), \pm\left(\frac{1}{4}, \frac{3}{4}, \frac{1}{4},-\frac{1}{4}\right), \pm\left(\frac{1}{2},-\frac{1}{2}, \frac{1}{2}, \frac{1}{2}\right), \pm\left(\frac{1}{4},-\frac{1}{4}, \frac{3}{4}, \frac{1}{4}\right), \\ \pm\left(-\frac{1}{2}, \frac{1}{2}, \frac{1}{2}, \frac{1}{2}\right), \pm\left(-\frac{1}{4}, \frac{1}{4}, \frac{1}{4}, \frac{3}{4}\right), \pm\left(\frac{1}{4},-\frac{1}{4}, \frac{1}{4}, \frac{3}{4}\right), \pm\left(-\frac{1}{4}, \frac{1}{4}, \frac{3}{4}, \frac{1}{4}\right), \\ \pm\left(\frac{3}{4},-\frac{1}{4}, \frac{1}{4}, \frac{1}{4}\right), \pm\left(\frac{1}{4}, \frac{1}{4}, \frac{3}{4},-\frac{1}{4}\right), \pm\left(\frac{1}{4}, \frac{1}{4},-\frac{1}{4}, \frac{3}{4}\right), \pm\left(-\frac{1}{4}, \frac{3}{4}, \frac{1}{4}, \frac{1}{4}\right), \\ \pm\left(\frac{1}{4}, \frac{1}{4}, \frac{1}{4}, \frac{1}{4}\right), \pm\left(\frac{1}{4}, \frac{1}{4}, \frac{1}{4},-\frac{3}{4}\right), \pm\left(\frac{1}{4}, \frac{1}{4},-\frac{3}{4}, \frac{1}{4}\right), \pm\left(\frac{1}{4}, \frac{1}{4},-\frac{1}{4},-\frac{1}{4}\right), \\ \pm\left(\frac{1}{4},-\frac{3}{4}, \frac{1}{4}, \frac{1}{4}\right), \pm\left(-\frac{3}{4}, \frac{1}{4}, \frac{1}{4}, \frac{1}{4}\right), \pm\left(\frac{1}{4},-\frac{1}{4}, \frac{1}{4},-\frac{1}{4}\right), \pm\left(\frac{1}{4},-\frac{1}{4},-\frac{1}{4}, \frac{1}{4}\right)\end{array}\right\}$ and $P_{1}+$ $P_{2}+P_{3}-\left|P_{4}\right|+P_{1} P_{2} P_{3}-P_{1} P_{2}\left|P_{4}\right|-P_{1} P_{3}\left|P_{4}\right|-P_{2} P_{3}\left|P_{4}\right|=\mathbf{0}$.

where $i \neq j, i \neq k, i \neq l, j \neq k, j \neq l, k \neq l$ and $i, j, k, l=1,2,3,4$.

\section{Conclusion}

In this work, an algorithm that gives the characterization of the linear combinations of finitely many diagonalizable matrices that mutually commute is proposed. The algorithm that takes the spectra of the matrices in the linear combination as inputs gives the characterization of the linear combination as output by solving the linear systems whose coefficients are taken from the spectra of the matrices. Every characteristic of the characterization is obtained by combining the results which have a permutation between their solutions or the results whose solutions are minus times of each other. Mathematica 8.0 notebook implementation codes 
are also provided (see DAS). Hence, the problems of characterizing the linear combinations of special matrices that mutually commute have become solvable by means of computers, and thus the results of these kinds problems now can be obtained for linear combinations of as many matrices as required. Moreover, the problems of cubicity of linear combibations of two commuting cubic matrices, quadripotency of linear combinations of two commuting quadripotent matrices, tripotency of linear combinations of three tripotent matrices that mutually commute, and tripotency of linear combinations of four involutive matrices that mutually commute are solved by utilizing the algorithm. These problems have been chosen because of the shortness of the presentation of their results. It is clear that other similar problems can also be solved by utilizing the algorithm. Some open problems with the number of linear systems that need to be solved are listed in Table 3 .

Table 3: Summary of open problems

\begin{tabular}{ll}
\hline The Problems & $\begin{array}{l}\text { The number of linear systems that } \\
\text { need to be solved }\end{array}$ \\
\hline 1) Quadraticity of linear combinations of & $\left(\begin{array}{c}8 \\
1\end{array}\right)+\left(\begin{array}{c}8 \\
2\end{array}\right)+\left(\begin{array}{c}8 \\
3\end{array}\right)=92$ \\
three quadratic matrices that mutually com- & \\
mute & \\
2) Cubicity of linear combinations of three cu- & $\left(\begin{array}{c}27 \\
1\end{array}\right)+\left(\begin{array}{c}27 \\
2\end{array}\right)+\left(\begin{array}{c}27 \\
3\end{array}\right)=3303$ \\
bic matrices that mutually commute & \\
3) Quadripotency of linear combinations of & $\left(\begin{array}{c}63 \\
1\end{array}\right)+\left(\begin{array}{c}63 \\
2\end{array}\right)+\left(\begin{array}{c}63 \\
3\end{array}\right)=41727$ \\
three quadripotent matrices that mutually & \\
commute & \\
4) Quadraticity of linear combinations of four & $\left(\begin{array}{c}16 \\
1\end{array}\right)+\left(\begin{array}{c}16 \\
2\end{array}\right)+\left(\begin{array}{c}16 \\
3\end{array}\right)+\left(\begin{array}{c}16 \\
4\end{array}\right)=2516$ \\
quadratic matrices that mutually commute & \\
5) Tripotency of linear combinations of four & $\left(\begin{array}{c}40 \\
1\end{array}\right)+\left(\begin{array}{c}40 \\
2\end{array}\right)+\left(\begin{array}{c}40 \\
3\end{array}\right)+\left(\begin{array}{c}40 \\
4\end{array}\right)=102090$ \\
tripotent matrices that mutually commute & \\
6) Cubicity of linear combinations of four cu- & $\left(\begin{array}{c}81 \\
1\end{array}\right)+\left(\begin{array}{c}81 \\
2\end{array}\right)+\left(\begin{array}{c}81 \\
3\end{array}\right)+\left(\begin{array}{c}81 \\
4\end{array}\right)=1752381$ \\
bic matrices that mutually commute & $\left(\begin{array}{c}255 \\
1\end{array}\right)+\left(\begin{array}{c}255 \\
2\end{array}\right)+\left(\begin{array}{c}255 \\
3\end{array}\right)+\left(\begin{array}{c}255 \\
4\end{array}\right)=$ \\
7) Quadripotency of linear combinations of \\
four quadripotent matrices that mutually & 174825280 \\
commute & \\
\hline
\end{tabular}

The first five of the problems are also solved with the help of the algorithm. Their results are given as program outputs (see DAS). To corroborate the algorithm, some problems in the literature, such as idempotency of linear combinations of two idempotent matrices [2], tripotency of linear combinations of two commuting tripotent matrices [5], idempotency of linear combinations of three idempotent matrices, two of which are commuting [7], and tripotency of a linear combination of two involutory matrices and a tripotent matrix that mutually commute [30], are reconsidered via the algorithm. Their results are given as outputs by comparing them with the results in the corresponding articles (see DAS).

Acknowledgment: The authors are very grateful to the anonymous referees for their constructive comments and suggestions.

Data Availability Statement: Mathematica 8.0 codes of the algorithm and the data that support the findings of this study are available in figshare repository at https://doi.org/10.6084/m9.figshare.14572713.v1.

\section{References}

[1] S.L. Adler, Quaternionic Quantum Mechanics and Quantum Fields, Oxford University Press Inc., New York, (1995). 
[2] J.K. Baksalary, O.M. Baksalary, Idempotency of linear combinations of two idempotent matrices, Linear Algebra Appl., 321, 3-7 (2000).

[3] J.K. Baksalary, O.M. Baksalary, On linear combinations of generalized projectors, Linear Algebra Appl., 388, 17-24 (2004).

[4] J.K. Baksalary, O.M. Baksalary, When is a linear combination of two idempotent matrices the group involutory matrix?, Linear Multilinear Algebra, 54(6), 429-435 (2006).

[5] J.K. Baksalary, O.M. Baksalary, H. Özdemir, A note on linear combinations of commuting tripotent matrices, Linear Algebra Appl., 388, 45-51 (2004).

[6] J.K. Baksalary, O.M. Baksalary, G.P.H. Styan, Idempotency of linear combinations of an idempotent matrix and a tripotent matrix, Linear Algebra Appl., 354, 21-34 (2002).

[7] O.M. Baksalary, J. Benítez, Idempotency of linear combinations of three idempotent matrices, two of which are commuting, Linear Algebra Appl., 424, 320-337 (2007).

[8] O.M. Baksalary., J. Benítez, On linear combinations of two commuting hypergeneralized projectors, Comput. Math. Appl., 56, 2481-2489 (2008).

[9] B. Baldessari, The distribution of a quadratic form of normal random variables, Ann. Math. Statist., 38, 1700-1704 (1967).

[10] A. Ben-Israel, T.N.E. Greville, Generalized Inverses: Theory and Applications, Springer-Verlag New York, (2003).

[11] J. Benítez, N. Thome, Idempotency of linear combinations of an idempotent matrix and a $t$-potent matrix that commute, Linear Algebra Appl., 403, 414-418 (2005).

[12] J. Benítez, N. Thome, Characterizations and linear combinations of $k$-generalized projectors, Linear Algebra Appl., 410, 150159 (2005).

[13] J. Benítez, N. Thome, $\{k\}$-group periodic matrices, SIAM J. Matrix Anal. Appl., 28, 9-25 (2006).

[14] J. Benítez, N. Thome, Idempotency of linear combinations of an idempotent matrix and a $t$-potent matrix that do not commute, Linear Multilinear Algebra, 56, 679-687 (2008).

[15] D.S. Bernstein, Matrix Mathematics, Theory, Facts, and Formulas, 2nd ed., Princeton U.P., New Jersey, (2009).

[16] R. Bru, N. Thome, Group inverse and group involutory matrices, Linear Multilinear Algebra, 45, $207-218$ (1998).

[17] C.Y. Deng, D.S. Cvetković-Ilić, Y. Wei, Properties of the combinations of commutative idempotents, Linear Algebra Appl., 436, 202-221 (2012).

[18] F.A. Graybill, Matrices with Applications in Statistics, second edition. Wadsworth, Belmont, (1983).

[19] J. Groß, G. Trenkler, Generalized and hypergeneralized projectors, Linear Algebra Appl., 264, 463-474 (1997).

[20] N.J. Higham, Functions of Matrices, SIAM, Philadelphia, (2008).

[21] R.A. Horn. and C.R. Johnson, Matrix Analysis 2nd ed., Cambridge U.P., Cambridge, (2013).

[22] E. Kişi, H. Özdemir, A combinatorial method for determining the spectrum of linear combinations of finitely many diagonalizable matrices that mutually commute, Math. Commun., 23, 61-78 (2018).

[23] X. Liu, L. Wu, J. Benítez, On linear combinations of generalized involutive matrices, Linear Multilinear Algebra, 59, 1221-1236 (2011).

[24] H. Özdemir, M. Sarduvan., A.Y. Özban, N. Güler, On idempotency and tripotency of linear combinations of two commuting tripotent matrices, Appl. Math. Comput., 207, 197-201 (2009).

[25] M. Sarduvan, H. Özdemir, On linear combinations of two tripotent, idempotent, and involutive matrices, Appl Math. Comput. 200, 401-406 (2008).

[26] M. Tošić, On some linear combinations of commuting involutive and idempotent matrices, Appl. Math. Comput., 233 103-108 (2014).

[27] M. Uç, H. Özdemir, A.Y. Özban, On the quadraticity of linear combinations of quadratic matrices, Linear Multilinear Algebra, 63, 1125-1137 (2015).

[28] Y.Wu, $K$-Potent matrices-construction and applications in digital image encryption, in: Recent Advances in Applied Mathematics, AMERICAN-MATH'10 Proceedings of the 2010 American Conference on Applied mathematics, USA, $455-460$ (2010).

[29] C. Xu, On the idempotency, involution and nilpotency of a linear combination of two matrices, Linear Multilinear Algebra, 63, 1664-1677 (2015).

[30] C. Xu, R. Xu, Tripotency of a linear combination of two involutory matrices and a tripotent matrix that mutually commute, Linear Algebra Appl., 437, 2091-2109 (2012). 